\title{
Level 1 algorithms for TANSO on GOSAT: processing and on-orbit calibrations
}

\author{
A. Kuze ${ }^{1}$, H. Suto ${ }^{1}$, K. Shiomi ${ }^{1}$, T. Urabe ${ }^{1}$, M. Nakajima ${ }^{1}$, J. Yoshida ${ }^{2}$, T. Kawashima ${ }^{2}$, Y. Yamamoto ${ }^{3}$, F. Kataoka ${ }^{4}$, \\ and H. Buijs ${ }^{5}$ \\ ${ }^{1}$ Japan Aerospace Exploration Agency, Tsukuba-city, Ibaraki, Japan \\ ${ }^{2}$ NEC Toshiba Space Systems, Ltd., Fuchu, Tokyo, Japan \\ ${ }^{3}$ NEC Informatec Systems, Ltd., Kawasaki-City, Kanagawa, Japan \\ ${ }^{4}$ Remote Sensing Technology Center of Japan, Tsukuba-city, Ibaraki, Japan \\ ${ }^{5}$ ABB, Inc., Quebec-city, Quebec, Canada \\ Correspondence to: A. Kuze (kuze.akihiko@jaxa.jp)
}

Received: 5 February 2012 - Published in Atmos. Meas. Tech. Discuss.: 24 April 2012

Revised: 18 September 2012 - Accepted: 19 September 2012 - Published: 19 October 2012

\begin{abstract}
The Thermal And Near infrared Sensor for carbon Observation Fourier-Transform Spectrometer (TANSOFTS) onboard the Greenhouse gases Observing SATellite (GOSAT) (nicknamed "Ibuki") has been providing global space-borne observations of carbon dioxide $\left(\mathrm{CO}_{2}\right)$ and methane $\left(\mathrm{CH}_{4}\right)$ since 2009. In this paper, we first describe the version V150.151 operational Level 1 algorithms that produce radiance spectra from the acquired interferograms. Second, we will describe the on-orbit characteristics and calibration of TANSO-FTS. Overall function and performance such as signal to noise ratio and spectral resolution are within design objectives. Correction methods of small onorbit degradations and anomalies, which have been found since launch, are described. Lastly, calibration of TANSO Cloud and Aerosol Imager (TANSO-CAI) are summarized.
\end{abstract}

\section{Introduction}

\subsection{Overview}

The Greenhouse gases Observing SATellite (GOSAT) was successfully launched into a sun-synchronous orbit on 23 January 2009 to monitor global distributions of carbon dioxide $\left(\mathrm{CO}_{2}\right)$ and methane $\left(\mathrm{CH}_{4}\right)$. GOSAT carries two instruments, namely, the Thermal And Near infrared Sensor for carbon Observation Fourier-Transform Spectrometer (TANSO-FTS) and the Cloud and Aerosol Imager (TANSO-
CAI). TANSO-FTS measures reflected solar radiance in the oxygen $\left(\mathrm{O}_{2}\right)$ A band region at $0.76 \mu \mathrm{m}$ (Band 1) and in the weak and strong $\mathrm{CO}_{2}$ bands at $1.6 \mu \mathrm{m}$ (Band 2) and $2.0 \mu \mathrm{m}$ (Band 3) respectively, and also $\mathrm{CH}_{4}$ bands at $1.67 \mu \mathrm{m}$ (Band 2) all with two orthogonal linear polarizations, designated "P" and "S". TANSO-FTS also measures spectral radiance in the thermal IR (Band 4). TANSO-CAI has four spectral bands, namely, $0.380 \mu \mathrm{m}$ (Band 1), $0.674 \mu \mathrm{m}$ (Band 2), $0.870 \mu \mathrm{m}$ (Band 3), and $1.60 \mu \mathrm{m}$ (Band 4) to determine clear soundings of the TANSO-FTS measurements and to provide cloud and aerosol optical properties.

GOSAT is a joint project of the Japan Aerospace Exploration Agency (JAXA), Ministry of the Environment (MOE) and the National Institute for Environmental Studies (NIES). JAXA is responsible for producing the Level 1A (raw interferogram) and the Level 1B (radiance spectra) products of TANSO-FTS and the Level 1A (raw digital image) product of TANSO-CAI. NIES provides the Level $2\left(\mathrm{CO}_{2}\right.$ and $\mathrm{CH}_{4}$ concentrations from Level 1B spectra), the Level 3 (global distribution of $\mathrm{CO}_{2}$ and $\mathrm{CH}_{4}$ concentrations by interpolating the Level 2 products), and the Level 4 (net $\mathrm{CO}_{2}$ sources and sinks) products of TANSO-FTS and the Levels 1B (radiance), 1B+ (spatially resampled data), 2 (with cloud flag), and 3 (global distribution) products of TANSO-CAI. These products are provided in the Hierarchical Data Format 5 (HDF5) format except for the FTS Level 4 product, which is saved in the Network Common Data Form (NetCDF) format and in text. The TANSO-FTS data level, product, format, 
TANSO-FTS

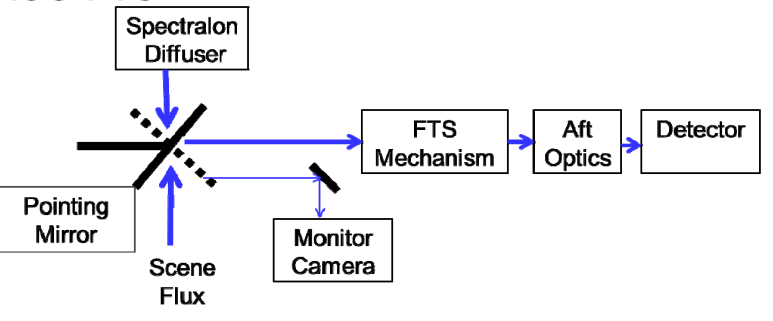

TANSO-CAI

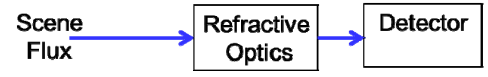

Fig. 1. The schematics of the TANSO-FTS and CAI optics modules.

and responsible institute are summarized in Table 1. All signals (Level 0) transmitted from the GOSAT satellite are received at Kongsberg Satellite Services AS (KSAT), Norway and Earth Observation Center (EOC), Japan and transmitted to Tsukuba Space Center (TKSC), Japan. Thereafter, the TANSO-FTS Levels 1A and 1B data and the TANSO-CAI Level 1A data are produced at TKSC. Usually the Level 1 data is transferred to NIES and other users such as the National Aeronautics and Space Administration (NASA) in the United States and the European Space Agency (ESA) within $5 \mathrm{~h}$ after the initial observation on orbit. The Level 1B data together with all the necessary calibration and correction data and the higher data products produced in NIES are available at the NIES GOSAT User Interface Gateway (GUIG). Data of a monitor camera (CAM) (see Fig. 1) are not processed as a Level 1 product because it was originally installed to check pointing mirror alignment onboard after launch, especially the difference between the actual viewing position and the calculated value from the resolver of the pointing mirror mechanism. We describe here the data processing algorithms of the TANSO Levels 1A and 1B. The details of the Level 2 algorithms are described in Yoshida et al. (2011) for TANSOFTS short wave infrared (SWIR) bands, Saitoh et al. (2009) for the thermal infrared (TIR) band, and Ishida et al. (2009, 2011) for TANSO-CAI.

\subsection{TANSO Instruments}

The schematics of the TANSO-FTS and CAI optics modules are illustrated in Fig. 1. TANSO-FTS has many functions and operational modes. The two-axis pointing mechanism has pointing and image motion compensation functions, allowing precise viewing of the Earth and the calibration sources. In nominal observation mode, the pointing mirror views predefined grid points of the Earth close to nadir as illustrate in Fig. 2. In the sun-glint observation and special target modes, the pointing mirror views the specular reflection points over the ocean and requested observation points, respectively. The FTS scan mechanism sweeps the optical path difference (OPD) of the interferometer. The per-
Table 1. TANSO-FTS data product format. Data level, data product, data format, and responsible institution.

\begin{tabular}{|c|c|c|c|}
\hline $\begin{array}{l}\text { Data } \\
\text { level }\end{array}$ & $\begin{array}{l}\text { Data } \\
\text { product }\end{array}$ & $\begin{array}{l}\text { Data } \\
\text { format }\end{array}$ & $\begin{array}{l}\text { Responsible } \\
\text { institute }\end{array}$ \\
\hline $1 \mathrm{~A}$ & Raw interferograms & HDF & JAXA \\
\hline 1B & Spectral radiance & $\mathrm{HDF}$ & JAXA \\
\hline 2 & $\begin{array}{l}X_{\mathrm{CO}_{2}} \text { and } X_{\mathrm{CH}_{4}} \\
\text { soundings }\end{array}$ & $\mathrm{HDF}$ & NIES \\
\hline 3 & $\begin{array}{l}\text { Global distribution } \\
\text { of } X_{\mathrm{CO}_{2}} \text { and } X_{\mathrm{CH}_{4}}\end{array}$ & $\mathrm{HDF}$ & NIES \\
\hline 4 & $\mathrm{CO}_{2}$ sources/sinks & NetCDF & NIES \\
\hline
\end{tabular}

formance of these two mechanisms on orbit has been carefully characterized and is described in Sects. 2.3.3 and 3.4. TANSO-CAI is a much simpler instrument. It has no mechanical moving parts and no onboard calibrator. It views a wide swath of $910 \mathrm{~km}$ and acquires a global image in 3 days. The spectral and geometric performance of TANSO-CAI on orbit is assumed to be stable. During the daytime both SWIR and TIR of TANSO-FTS and TANSO-CAI data are acquired. During the nighttime only TANSO-FTS TIR data are acquired. Overall the radiometric responses of both TANSOFTS and CAI are the combination of the optical efficiency, detector response, and the gain of the analog circuits. The onorbit response changes have been monitored for three years and their characterization is described in this paper. Details related to each instrument design, prelaunch hardware performance, and on-orbit operations were described in Kuze et al. (2009).

\subsection{GOSAT operation}

The function and performance of all systems were verified to meet operating standards during the initial threemonth checkout phase after launch (mid-January to midApril 2009). In the second three-month phase (May to July 2009), the initial calibration was performed. During this period, the first vicarious calibration campaign was performed and the responses of TANSO-FTS and CAI were calibrated (Kuze et al., 2011a). The nominal operation phase started in July 2009. More than three years have passed since launch, and the overall functions and performances are within design objectives. All measured radiance spectra of the TANSO-FTS SWIR and TIR bands and columnaveraged dry air mole fractions (the Level 2 product) of $\mathrm{CO}_{2}$ and $\mathrm{CH}_{4}\left(X_{\mathrm{CO}_{2}}\right.$ and $X_{\mathrm{CH}_{4}}$, respectively) retrieved from the SWIR bands are currently available to the public from NIES. In addition to the standard GOSAT Level 2 product provided by NIES, several other working groups have derived $X_{\mathrm{CO}_{2}}$ and $X_{\mathrm{CH}_{4}}$ from the TANSO Level 1 data using their own processors (Butz et al., 2011; Parker et al., 2011; O'Dell et al., 2012; Crisp et al., 2012). In addition to the greenhouse gases, chlorophyll fluorescence has been measured from 


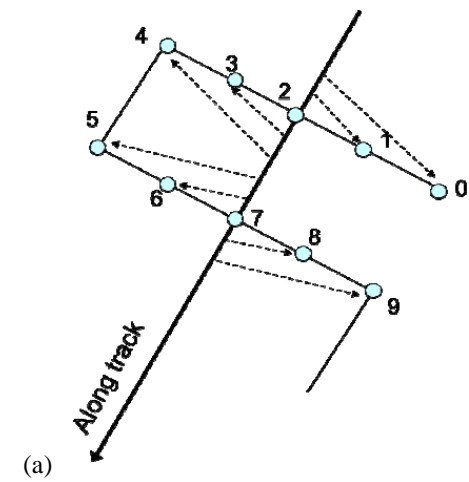

(b)
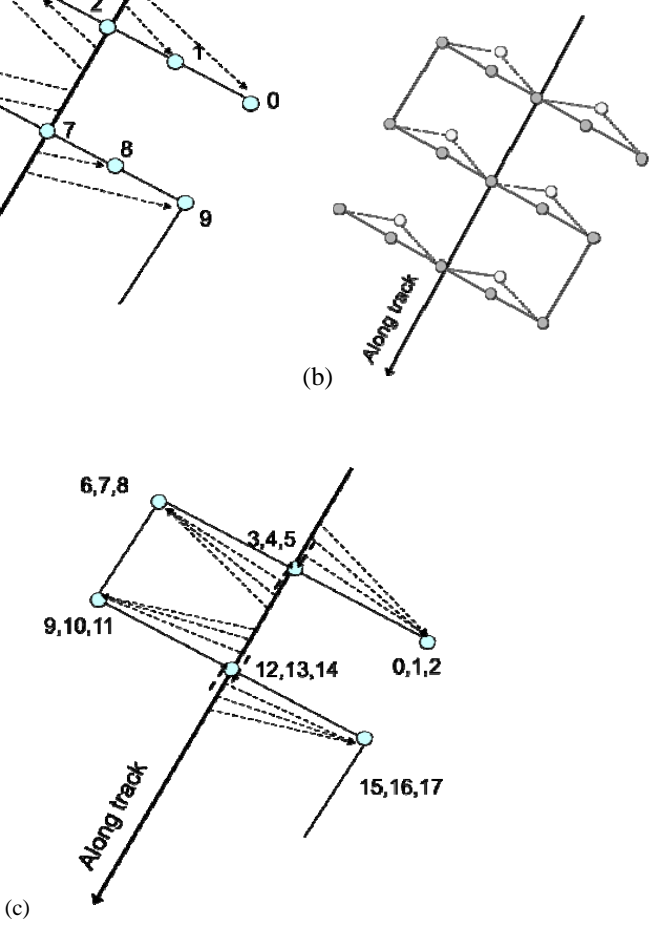

Fig. 2. Grid observation patterns: (a) 5-point cross-track scan mode (b) a $\mathrm{M}$ shape grid overwritten on 5-point cross-track scan mode, and (c) 3-point cross-track scan mode.

space for the first time using high-spectral-resolution Fraunhofer line spectra by Joiner et al. $(2011,2012)$, Frankenberg et al. (2011a, b), and Guanter et al. (2012).

GOSAT has a 3-day revisit orbit cycle and a 12-day operation cycle. Three different operational patterns have been employed to insert target observations into nominal grid observations. These are pattern A, consisting of limited calibration and validation site observations; pattern $\mathrm{B}$, consisting of sun glint over the ocean near the sun's declination as well as target observations requested by registered researchers responding to research announcements (RA); and pattern C, consisting of sun glint and limited calibration and validation site observations. Each pattern is maintained for three days and the repeated order of the patterns is A, B, C, and B. The detailed operation modifications in chronological order were described in Kuze et al. (2011b).

Several anomalies were found in on-orbit operation of TANSO-FTS and the operation was modified to avoid performance degradation (Kuze et al., 2011c). One of the major anomalies is the zero path difference (ZPD) shift, which degrades the spectral resolution and needs to be corrected. ZPD is the center of the interferogram. The FTS scan arm is servo-controlled using monochromatic laser fringes. This achieves uniform-speed scanning within the OPD range followed by rapid reversal of the scan-arm motion (turnaround).
The fringe count is also used to trigger the turnaround of the scan mechanism at precisely repeatable scan distance from ZPD. This is done by up and down counting according to scan direction. During turnaround, both FTS scan and pointing mirror are activated simultaneously. A combination of microvibrations caused by the pointing motion, a slower speed scan at turnaround and in an electro-magneticnoise environment, has reduced the reliability of the fringe count which occasionally causes the controller of the FTSmechanism to miss count the laser fringes at the turnaround position and consequently causes the ZPD position to shift gradually. Now periodically the ZPD position is reset by uploading a fringe shift command from the ground. The shifted fringe number is recorded in the Level 1B product.

The other major anomaly is a pointing bias of the pointing mirror, which may be due to a bias of the angular resolver (an angular position sensor) or insufficient electrical power to properly drive the along track (AT) motor. Also, within the AT motion range, there are several "dead band" zones, where the AT motion becomes unstable or even occasionally settles down in an incorrect position. The 2nd and 4th of the original five cross-track (CT) points shown in Fig. 2a are close to dead band angles and they have large biases: in some cases they can be detected by the resolver and then can be geometrically corrected. Instead of the nominal 5-point grid, an $\mathrm{M}$ shape pattern as illustrated in Fig. 2b was introduced between 26 June 2009 and 31 July 2010. This is to avoid the dead band areas and to minimize the pointing anomalies. The exact pointing locations of the $\mathrm{M}$ shape can be retrieved in the Level 1B geolocation data. With a simple 3-point crosstrack pattern as shown in Fig. 2c dead band zones are also avoided. Therefore, a simpler 3-point cross-track scan mode has been employed more recently. The trend of the bias and the geometric collection method using camera data are to be described later.

Between launch and July 2010, for the 5-point cross-track scan mode, interferograms had been acquired every $4.45 \mathrm{~s}$ ( $4 \mathrm{~s}$ for interferogram acquisition and $0.45 \mathrm{~s}$ for turnaround). This resulted in $150 \mathrm{~km}$ spacing between grid observations and a total of 56000 soundings globally every three days. Since August 2010, the grid observation pattern has changed from the 5-point cross-track scan mode to the 3-point crosstrack scan mode with $260 \mathrm{~km}$ spacing. Implementation of a longer turnaround time of $0.6 \mathrm{~s}$ probably decreased the ZPD shift rate as well. The sun glint observation uses the image motion compensation (IMC) to minimize the input intensity fluctuation and to reduce the cloud contamination.

Thus, so far, owing much to the careful satellite and sensors operation from the ground, the overall function of TANSO-FTS has been stable. The radiometric, geometric, spectrometric, and polarimetric performances have been good owing to the modification of the Level 1 algorithm and proper calibrations. The Level 1 algorithm and processing flows to create radiance spectra from acquired interferograms are described in Sect. 2. In Sect. 3, the performance 
characterization of on-orbit, onboard and vicarious calibrations are presented. Lastly, TANSO-CAI characterization and calibration are described in Sect. 4.

\section{TANSO-FTS Level 1 algorithm}

In this section, the version V150.151 TANSO-FTS Level 1 algorithm is described. The algorithm has been updated several times since launch. The versions in chronological order were described in Kuze et al. (2011b).

All the unprocessed numerical form interferograms are saved as Level 1A data. The payload correction data from the satellite bus and telemetry data of TANSO-FTS are added in both Levels $1 \mathrm{~A}$ and $1 \mathrm{~B}$ products together with the measured data. These data include the time stamp, the satellite and sun position, the geolocation, the AT and CT angles of the pointing mirror, the detector temperature, the blackbody temperature, and gain level, all of which are necessary for the data processing and analysis. The time stamp in the data shows the start of the interferogram acquisition. The satellite position is interpolated by using the payload correction data, which is transmitted from the satellite every second. The AT, $\mathrm{CT}$, solar angles, and the geometry information are acquired at the start of the interferogram acquisition as well.

\subsection{Procedures}

Interferograms of each band (two linear polarizations of bands 1,2, and 3 and scalar band 4) are processed independently. TANSO-FTS bands 1, 2 and 3 (SWIR) are processed as follows:

Step S1: Saturation detection

Step S2: Correction of spikes caused by cosmic rays hitting the detector

Step S3: Scan speed instability correction (applied for the Band 1 medium gain only)

Step S4: Intensity variation correction (bands 2 and 3 only)

Step S5: OPD sampling interval non-uniformity correction

Step S6: Direct current (DC) subtraction and ZPD detection

Step S7: Inverse fast Fourier transform (IFFT)

Step S8: Phase correction

Step S9: Analog circuit nonlinearity correction.

TANSO-FTS Band 4 (TIR) is processed as follows:

Step T1: Saturation detection

Step T2: Nonlinearity correction of the Band 4 photo conductive (PC) - mercury cadmium telluride (MCT) detector
Step T3: Correction of spikes caused by cosmic rays hitting the detector

Step T4: OPD sampling interval non-uniformity correction

Step T5: ZPD detection

Step T6: IFFT

Step T7: Complex radiometric calibration and deep space (DS) view obscuration correction

Polarization correction of the pointing mirror.

A detailed flow diagram is shown in Fig. 3 and each process is explained in the following subsections.

\subsection{Introductory remarks}

On the TANSO-FTS, interferograms are measured on both sides of the ZPD position providing "double-sided" interferograms. Furthermore the three SWIR bands are quite narrow compared to their center wavelength in order to minimize unnecessary photon noise. At the same time the interferograms are sampled at high OPD density compared with the bandwidth of each band. There is no onboard data compression. The result is that the out of band zero parts of the resulting spectra as well as the imaginary component of the spectrum provide a sensitive check on saturation, nonlinearity, and OPD sampling uniformity.

\subsection{Corrections}

\subsubsection{Saturation, low frequency fluctuation, and spike detection, and correction (Steps S1, S2, T1, and T3)}

First, saturated interferograms are screened. Saturation at the ZPD of interferograms occurs mainly in the pre-amplifier and analog-to-digital converter (ADC). As the outputs of the preamplifiers are phase delayed, the saturation at the input cannot be explicitly detected and corrected in real time. Indirect evidence of saturation can be obtained from out of band spectral intensities. Low frequency fluctuations seen on some interferograms are generated by the pointing mirror mechanism's unstable settling down over nonuniform targets. There is the possibility of saturation when the interferogram reconstructed for screening purpose from low frequency components is additive to the normal ZPD signal or the digital number exceeds 65400 , which is slightly lower value than the actual dynamic range providing some margin. Independent of saturation, interferograms that have jumped due to a gain change or have significant fluctuations are also screened. Fortunately, there have been no cosmic rays detected since launch. But high energy particles also create spikes. These spikes are screened and a quality flag is attached to the Level 1B data. Fluctuations are corrected in the low frequency component correction process described in Sect. 2.3.4 and 


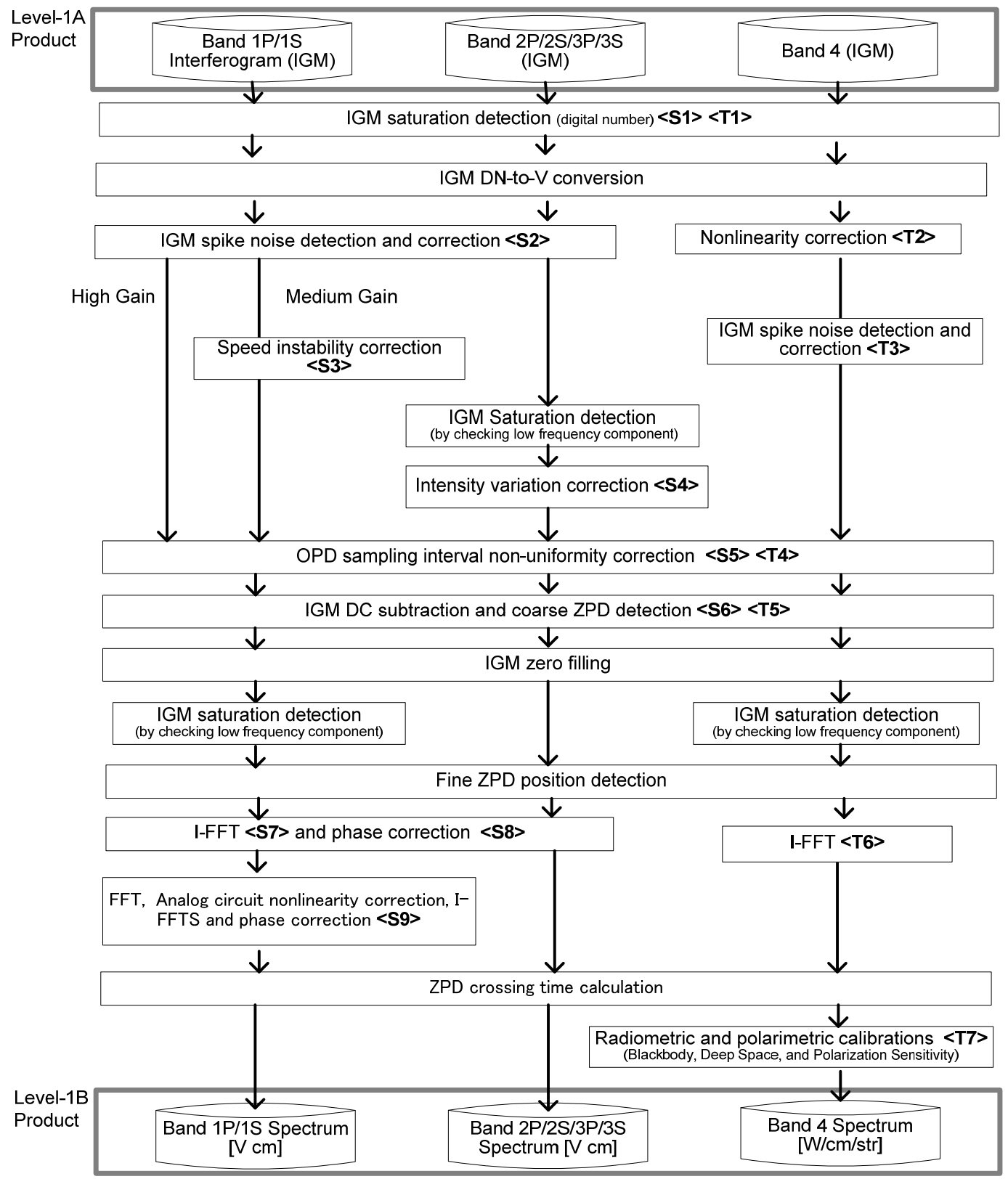

Fig. 3. The TANSO-FTS Level 1 data processing flow. Steps S1-S9 and T1-T5 are indicated in bold letters.

spikes are removed and filled with an estimated DC signal, which is the average of the interferogram levels acquired before and after the spike.

\subsubsection{Digitization and its nonlinearity}

Nonlinearity of the raw interferogram readout requires correction. There are three blocks in the signal electronics: namely the detector, analog circuit, and ADC. The Si detectors of Band 1 and InGaAs detectors of bands 2 and 3 are adequately linear. The Band 4 PC-MCT detector has nonlinearity, and its correction method is described in Sect. 2.3.7.
The analog circuit consists of the current-to-voltage converter (transimpedance amplifier), gain amplifier, and electric filters: their characteristics and correction are described in Sect. 2.3.6.

FTS has a wide dynamic range signal requiring high resolution 16-bit ADCs for all bands. With two gain levels, high and medium, an adequate numerical representation can be obtained for all scene conditions. However, when the surface albedo is very low, the number of ADC bits used is limited and quantization may have a small bias. In addition, dynamic range used for very low signal puts high demand on ADC linearity. ADC nonlinearity can be characterized by 


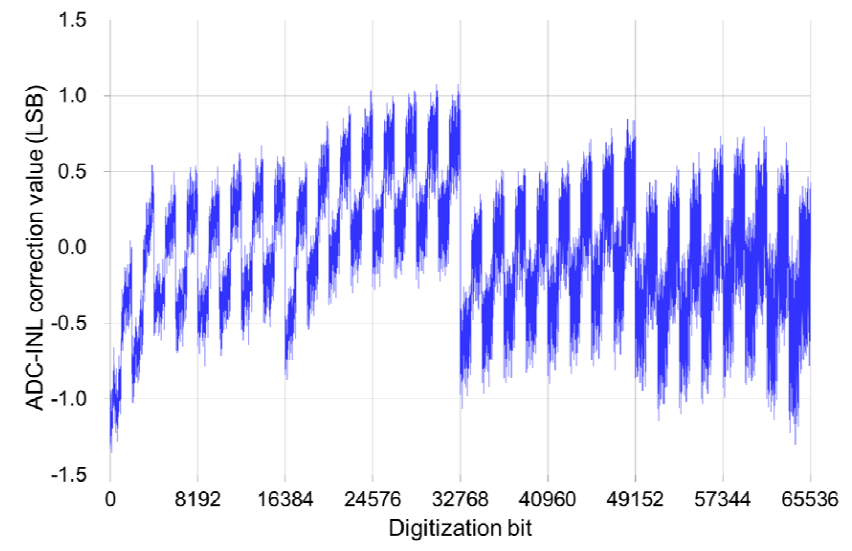

Fig. 4. ADC integral non-uniformity correction value retrieved from the measured data using the engineering model.

their differential nonlinearity (DNL) and integral nonlinearity (INL). A DNL larger than 1 bit becomes a missing code. By measuring DNL bit by bit, the INL of the entire dynamic range can be characterized. Figure 4 shows the INL correction value retrieved from measured data using the TANSOFTS engineering model. ADC nonlinearity produces spectral artifacts, which vary with the intensity level of the input flux. The 16-bit ADCs used in TANSO-FTS have the largest nonlinearity at the center of the dynamic range. The Band 1 signals are alternating current $(\mathrm{AC})$ coupled and the center bit is frequently used as OPD increases. Although INL of the entire dynamic range has been characterized, it has proven very difficult to correct the ADC nonlinearity particularly for low level AC sampled interferograms. In version V130.130 (the previous version of Level 1B processing) correction of the ADC nonlinearity was attempted. However in the case of low input signal, the resulting Level 1B products suffered from large artifacts. Therefore, the version V150.151 does not correct the ADC nonlinearity. As a result, in the case of very low signal flux, the Level 1B data of Band 1 may have a possible small bias.

\subsubsection{Correction of scan speed instability (Step S3)}

The phase delay mismatch between the metrology laser electronics and science channel degrades the robustness to scan speed instability and creates artifacts in the spectra. Only the medium gain of Band 1, which is used over high reflectance regions such as the Sahara, Arabian and Australian deserts and solar calibration, has a significant delay mismatch: group delays of high and medium gains are different and the delay matching is applied only to high gain. When speed instability is large, the Band 1 interferogram with the medium gain is not uniformly sampled. In the worst case, the sampling interval changes by about $\pm 0.6 \%$ and the spectral radiance changes by $\pm 1.5 \%$. The speed instability is caused by a combination of two sinusoidal microvibration sources, namely, a
$244 \mathrm{~Hz}$ from the satellite attitude control's Earth sensor and the $325.5 \mathrm{~Hz}$ resonant frequency of the FTS mechanism. Except for these two oscillations, the on-orbit mechanical environment is very quiet. Since the nominal scan speed is very constant, these two additional modulations to the interferogram have quite pure sinusoidal variations and can be corrected by retrieving their amplitudes and phases for each interferogram acquired with medium gain. As each interferogram is processed, these four parameters are estimated to minimize the out-of-band artifact spectra. The interferogram is then interpolated at the expected sampling positions but at the cost of additional computation resources in the ground data processing (Simon, 2008; Suto and Kuze, 2010). After applying the correction introduced in Version 110.110, the standard deviation of the Band 1 medium gain data has been significantly reduced to the noise level (Suto et al., 2011a).

\subsubsection{Correction of the intensity variation component (Step S4)}

The acquisition of both modulated and non-modulated portions of the DC coupled interferogram of bands 2 and 3 is performed in order to check the data quality. The metrology wavelength of TANSO-FTS is $1.31 \mu \mathrm{m}$. Even sampling the OPD at half the wavelength by means of up-and-down zerocrossings of laser fringes, the wavelength of Band 1 is still shorter than twice the sampling interval and hence Band 1 frequencies are aliased to low frequencies. Both optical and electrical filters are employed to minimize aliased signal and noise mixing. This also necessitates AC coupling of the signal to the ADC and hence preventing the data quality determination as is done for bands 2 and 3. Band 4 is also AC sampled to reduce the non-modulated background radiation. By checking the DC coupled interferogram, changes in the scene radiant intensity such as erratic intensity changes due to the pointing anomaly can be detected. DC coupled interferograms may also show low frequency variations which are indicative of pointing jitter and optical vignetting. These frequencies are typically lower than $500 \mathrm{~Hz}$ and much lower than the scene signal modulation of the interferometer which is of the order of $10 \mathrm{KHz}$. They have independent amplitude and phase and can be extracted from the interferogram. We remove the low frequency component by dividing an interferogram created from the low frequency components. This method is similar to the method applied to the FTS units of the Total Carbon Column Observing Network (TCCON) (Keppel-Aleks et al., 2007).

The largest contribution to the low frequency components is the optical vignetting. The FTS mechanism is based on the double-pendulum scanning mechanism principle and its effective optical throughput becomes slightly lower as OPD increases due to vignetting. This apodization effect is corrected, leaving the apodizations caused by the finite size of the field of view and the detector alignment offset from the optical axis. All the bands 2 and 3 data have vignetting and 


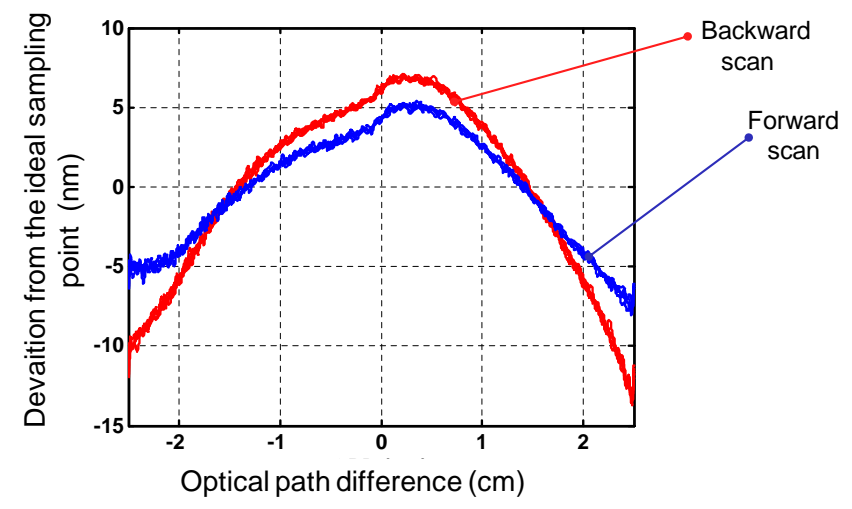

Fig. 5. Sampling interval non-uniformity as a function of OPD of the forward and backward scans.

are corrected. The field of view of TANSO-FTS is too large to permit realization of the full resolution for Band 1. The interferograms of Band 1 undergo strong self-apodization and as a result Band 1 has a different resolution compared with bands 2,3 , and 4 .

The second contribution is scene flux fluctuation due to the constant satellite sinusoidal vibration motion. The typical frequency is about $10 \mathrm{~Hz}$ and the possible source is the satellite's reaction wheel. The last contribution is the pointing mirror fluctuation, which occurs irregularly. About $20 \%$ of the total interferograms are affected by the fluctuation caused by the pointing mirror and have intensity modulation. We can detect these two fluctuations by checking the Band 2 interferograms. If the scene is spatially uniform, we cannot detect the pointing mirror fluctuations. At the same time, the spectra of the spatially uniform targets are not affected by these fluctuations.

\subsubsection{Correction of OPD sampling interval non-uniformity (Steps S5 and T4)}

The ideal interferogram of the scene flux would be acquired with equal sampling intervals of the laser fringes within the entire OPD range. The actual on-orbit interval decreases by only $25 \mathrm{~nm}$ from ZPD to maximum OPD. The change is not linear with OPD, and the forward and backward scans of the FTS mechanism are slightly different as shown in Fig. 5. The error caused by this non-uniformity is close to the noise level in the forward direction data but slightly larger in the backward scan data. The root cause is not well-known, but it is probably caused by the laser optical misalignment, asymmetry of the FTS scan-mechanism structure, and its electronic control imperfection. The deviation on orbit can be estimated by analyzing the onboard ILSF calibration laser data. As the deviation is reproducible on orbit, this non-uniformity is corrected by resampling the interferogram.

\subsubsection{Correction of Band 1 analog circuit nonlinearity (Step S9)}

The high gain of Band 1 uses a Chebyshev filter with much sharper gain peak and cutoff than the other bands to avoid aliasing of noise. Unfortunately, its gain is sensitive to the capacitance in the circuit, which varies with input voltage, temperature, and time. Apart from the high gain Band 1, all bands use Butterworth filters, which have flat responses. Changes in their feedback capacitance result only in small shifts in their cutoff. The nonlinearity induced by the inputvoltage change can be corrected using a 3rd order polynomial (Suto et al., 2011b). These coefficients are determined such that the out-of-band artifact spectra are minimized. The nonlinearity is a function of both the input voltage and the ZPD level. Thus, the nonlinearity is corrected after the phase correction as indicated in Fig. 3. We have prepared the 3rd-order-coefficient tables as a function of the ZPD voltage. Temperature dependency might cause seasonal and orbit phase changes and the time dependency might cause long-term changes. Band $1 \mathrm{P}$ has shown an especially larger change and these effects need further investigation. We replaced the capacitor of the engineering model with a temperature compensated one in the laboratory on the ground and then the artifact spectra were removed. These test results also indicate that our correction algorithm works well.

\subsubsection{Correction of TIR detector nonlinearity (Step T2)}

We use a photo-conductive PC-MCT detector, which has nonlinearity for strong signal input for Band 4 . The nonlinearity can be corrected using a 2 nd order polynomial. Assuming that nonlinearity characteristics do not change with time after launch, the coefficients can be determined from the well-characterized large photon input data under stable conditions in a vacuum chamber prior to launch. There are two ways to find the correction parameters. When the detector and its analogue electronics have nonlinearity, the largest signal at ZPD is distorted and artifact low frequency spectra are created. The correction coefficients are tuned such that interferograms filtered to have only low frequency components are flat near ZPD. The other method is to select the correction coefficients to fit the artifact shape at the edges of the band, i.e. $300-600 \mathrm{~cm}^{-1}$ and $2200-3500 \mathrm{~cm}^{-1}$. The latter method is applied as it has greater sensitivity to determine the coefficients. They are fine-tuned such that corrected signals of the out-of-band region are flat. The nonlinearity corrected data $V_{\text {NLcorrected }}$ is calculated by Eqs. (1) and (2) described below with measured raw AC components of the interferogram in voltage $V_{\mathrm{AC}}$ and DC voltage $V_{\mathrm{DC}}$. $V_{\mathrm{DC}}$ is the average of DC samples, which are monitored at 38 samples per interferogram. We subtract offset $V_{\mathrm{DCoffset}}$, which has a gradual increase since launch probably due to temperature change of the electric circuit. $V_{\text {DCoffset }}$ is estimated from the DS view 
data and modeled as a function of time since launch.

$V_{\text {Pamp }}=-\left(\left(V_{\mathrm{DC}}-V_{\mathrm{DCoffset}}\right) / g_{\mathrm{DC}}\right)-V_{\mathrm{AC}} / g_{\mathrm{AC}}$.

The gain factors $g_{\mathrm{DC}}$ and $g_{\mathrm{AC}}$ are 0.681 and 110.103 , respectively.

$V_{\text {NLcorrected }}=V_{\text {Pamp }}+a_{\text {nlc }} V_{\text {Pamp }}^{2}$.

$a_{\mathrm{nlc}}$ is the nonlinearity correction coefficient of 0.6056 .

\subsection{Inverse fast Fourier transform}

\subsubsection{ZPD detection and inverse fast Fourier transform (Steps S6, S7, T5, and T6)}

The ZPD position is determined by searching the center burst (peak) of the interferogram. The interferogram is then converted to the spectra with a prime factor IFFT. ZPD position of the SWIR bands is searched in every interferogram, assuming that the true ZPD is located near the center burst. On the other hand, for the TIR band, scene flux from the ground and atmosphere is close to the background radiation (mainly from the aft optics) and it can be difficult to detect the ZPD position, as the positive signal of input scene flux is cancelled by the negative signal of the radiation from the aft optics. Ideally, the ZPD position is calculated from the DS calibration when the background is dominant and we assume that it will not change between the DS calibrations, which typically occur in intervals of 10-25 min. However, when ZPD shift occurs, the ZPD has to be detected again such that the phase becomes spectrally flatter.

The number of interferogram data used for inverse Fourier transform is 76336. As many interferogram data are extracted as needed for data processing centered on ZPD. Based on the measured laser wavelength before launch, the exact maximum OPD are 1309.742/2 nm $\times \pm$ $38168= \pm 2.4995 \mathrm{~cm}$ for the primary laser source and $1309.688 / 2 \mathrm{~nm} \times \pm 38168= \pm 2.4994 \mathrm{~cm}$ for the secondary, which is slightly smaller than $\pm 2.5 \mathrm{~cm}$. Prime number-based combinations of $76545\left(=3^{7} \times 5 \times 7\right)$ for bands 1,2 , and 3 and of $38400\left(=2^{9} \times 3 \times 5^{2}\right)$ for Band 4 have been selected for the FFT to save computation time instead of using 76336 and 38168 and to minimize the resulting spectrum size instead of using $131072\left(=2^{17}\right)$ and $65536\left(=2^{16}\right)$. A zerofilling at both ends of the interferogram data should be carried out to fill the balance of the FFT input vector after ZPD shift correction is performed. The laser temperature is controlled at a nominal value of $25^{\circ}$ with variations smaller than the thermometer resolution of $0.7 \mathrm{mK}$. Therefore, the laser wavelength has been very stable and OPD has been constant on orbit. When the ZPD position is away from the center of an interferogram by more than 100 fringes, a quality (warning) flag is set and ZPD is placed at the center of the FFT input vector. If the detected ZPD position is away by more than 2000 fringes, interferogram data is processed assuming that ZPD detection fails and the center of the FFT vector lies on the center of the acquired entire fringes.

\subsubsection{Phase correction (Step S8)}

For SWIR data processing, electrical frequency characteristics, wavelength dependency of index-of-refraction, and the ZPD position difference smaller than a laser fringe determine the phase function. The phase functions of the SWIR bands have spectrally smooth structures. The phase can be retrieved from the ratio of the imaginary to the real spectra. The phase is corrected such that imaginary spectra represent the noise level without any spectral features. It is known that for a reliable phase reconstruction one has to use low resolution phase spectra. At highly absorbed lines, the phase is very noisy and if used at full resolution it will be overcorrected. By significantly reducing the spectral resolution of the phase determination a closer representation of the smooth phase structure is obtained. The reduced resolution phase is obtained with Gaussian apodization. For TIR data processing, the complex radiometric calibration and the phase correction are performed simultaneously.

\subsection{SWIR Level $1 \mathrm{~B}$ product}

The real part of the complex spectrum is the measured signal while the imaginary part should represent the noise level of the measurement. The raw spectrum is used to estimate signal-to-noise ratio (SNR) levels. The unit is output voltage per wavenumber $(\mathrm{V} \mathrm{cm})$. Subsequently, the spectral data with both real and imaginary parts are stored as the Level 1B data before converting to transmittance or spectral radiance. In addition, if degradation of the instrument optical efficiency or detector response is detected, it is possible to update the radiance conversion factor without changing the Level $1 \mathrm{~B}$ product. Response degradation is discussed in Sect. 3.1. Low frequency components are also stored as they include information on pointing fluctuations, target scene changes, and microvibrations.

\subsection{Complex radiometric calibration and corrections for TIR (Step T7)}

DS is a very cold target with a temperature of $3 \mathrm{~K}$ and its view data are equivalent to that of the background radiation from the TANSO-FTS optics. A blackbody source (BB) is used as the higher temperature standard. TIR spectral radiance is calibrated with these two on-orbit reference sources without using prelaunch calibration. In addition to the detector nonlinearity correction, two more fine corrections described below are needed: DS view obscuration and polarization.

Since both DS and BB calibrations, consisting of 2 sets of forward and backward data of a total of 8 interferograms, are scheduled frequently (twice during the daytime and 4 times during the nighttime per orbit), on-orbit optical efficiency degradation does not affect the radiometric accuracy. 
Spectral radiances after the complex calibration from DS and BB spectra as expressed in Eq. (3) are provided as the Level 1B data. The forward and backward scan spectra are calibrated independently using the better set of forward and backward DS and BB scan data out of two.

$B_{\mathrm{obs}}(v)=\frac{S_{\mathrm{obs}}(v, d)-\left(S_{\mathrm{DS}}(v, d)-\Delta S_{\mathrm{DS}}(v, d)\right)}{S_{\mathrm{BB}}(v, d)-\left(S_{\mathrm{DS}}(v, d)-\Delta S_{\mathrm{DS}}(\nu, d)\right)}$

$B_{\text {BBeffective }}(v)$

In the above equation, $S_{\mathrm{obs}}(\nu, d), S_{\mathrm{DS}}(\nu, d)$, and $S_{\mathrm{BB}}(\nu, d)$ denote measured spectra of nadir observation, DS, and BB, respectively. The symbols $v$ and $d$ are the wavenumber and scan direction of the FTS mechanism.

Due to the pointing bias described earlier, the DS view is estimated to be obstructed by $3 \%$ of the entire scene flux with the inner surface of the DS view hood of an estimated temperature of $250 \mathrm{~K}$. The DS view obscuration correction is described as follows:

$\Delta S_{\mathrm{DS}}=\gamma \frac{B\left(T_{\mathrm{DShood}}\right)}{B\left(T_{\mathrm{BB}}\right)}\left(S_{\mathrm{BB}}-S_{\mathrm{DS}}\right)$,

where $\gamma$ is the obscuration fraction and $T_{\mathrm{DShood}}$ and $T_{\mathrm{BB}}$ are the temperature of the DS view hood and BB. $B(T)$ is the calculated spectral radiance of temperature of $T$. $T_{\mathrm{DShood}}$ is an estimated value and $T_{\mathrm{BB}}$ is the blackbody temperature monitored with the onboard temperature sensors.

The effective spectral radiance $B_{\text {BBeffective is calculated }}$ with the equation below.

$\mathrm{B}_{\mathrm{BBeffective}}=\varepsilon B\left(T_{\mathrm{BB}}\right)+(1-\varepsilon) B\left(T_{\text {background }}\right)$.

In the version V150.151, we use the emissivity $\varepsilon$ of 1 assuming that the environment temperature $T_{\text {background }}$ is close to the blackbody temperature. More detailed correction by using measured emissivity, background-view factor from the blackbody and background temperature, is to be applied in the future.

The pointing mirror is viewing DS and $\mathrm{BB}$ calibration sources horizontally by rotating the mirror by about $90^{\circ}$, whereas the scene flux is acquired from nadir. The coating of the pointing mirror is optimized for SWIR and both the pointing mirror and the FTS optics have polarization sensitivity at TIR region. Lastly, the polarization effect is corrected by using the following relation between $B_{\text {obscorrected }}(v), B_{\text {obs }}(v)$, and $B_{\text {mirror }}(\nu)$ in case of nadir looking.

$$
\begin{aligned}
& B_{\text {obscorrected }}(\nu)=\frac{\left(\left(p_{2}^{2}+q_{2}^{2}\right)\left(p_{1}^{2}+q_{1}^{2}\right)-\left(p_{2}^{2}-q_{2}^{2}\right)\left(p_{1}^{2}-q_{1}^{2}\right)\right)}{\left(\left(p_{2}^{2}+q_{2}^{2}\right)\left(p_{1}^{2}+q_{1}^{2}\right)+\left(p_{2}^{2}-q_{2}^{2}\right)\left(p_{1}^{2}-q_{1}^{2}\right)\right)} B_{\mathrm{obs}}(\nu) \\
& +\frac{2\left(p_{2}^{2}-q_{2}^{2}\right)\left(p_{1}^{2}-q_{1}^{2}\right)}{\left(\left(p_{2}^{2}+q_{2}^{2}\right)\left(p_{1}^{2}+q_{1}^{2}\right)+\left(p_{2}^{2}-q_{2}^{2}\right)\left(p_{1}^{2}-q_{1}^{2}\right)\right)} B_{\text {mirror }}(v)+\Delta B_{\mathrm{bg}}(v)
\end{aligned},
$$

where $p_{1}^{2}$ and $q_{1}^{2}$ are optical efficiencies of two linear polarizations of the pointing mirror and $p_{2}^{2}$ and $q_{2}^{2}$ correspond to the FTS optics and the aft-optics. $B_{\text {mirror }}(v)$ is the radiation

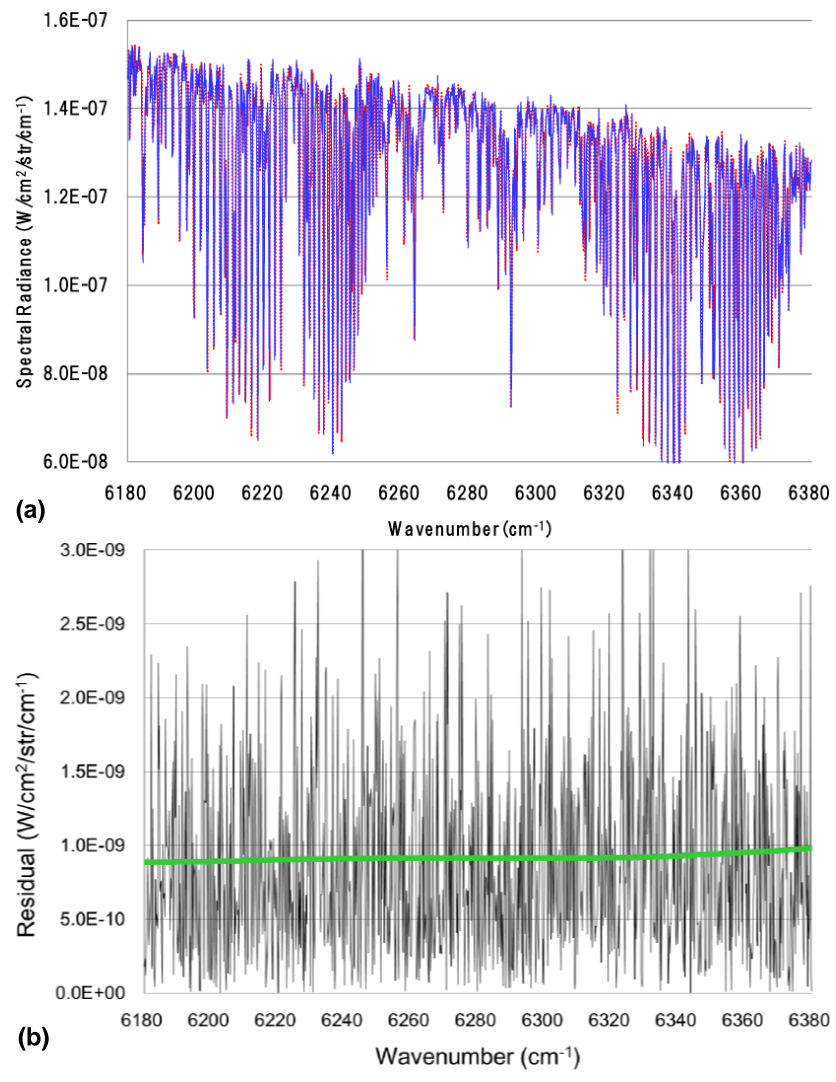

Fig. 6. (a) Measured $\mathrm{CO}_{2}$ spectrum (blue solid line) of the Level 1B V050.050 along with the modeled spectrum for the retrieval (red dotted line) and (b) the residual difference (black solid line) together with the noise level (bold green line) (courtesy of Y. Yoshida of NIES).

from the pointing mirror surface, which is estimated from the temperature telemetry data near the pointing mirror. $\Delta B_{\mathrm{bg}}(v)$ is the background radiation change between BB and DS calibrations, which is estimated from the orbit phase temperature variation model. $B_{\text {obscorrected }}(v)$ is the final Level 1B TIR products. A more general formulation is described in Mueller matrix in Sect. 3.5.

\subsection{Time data in Level $1 \mathrm{~B}$ product}

The time stamp of the telemetry data is the turnaround completion time $T_{\mathrm{TAT}}$, which corresponds to the start of interferogram acquisition. The interferogram near ZPD is the most informative and ZPD passing time is calculated using the following relationship:

$T_{\mathrm{ZPD}}=T_{\mathrm{TAT}}+T_{\mathrm{S}} \frac{X_{\mathrm{ZPD}}}{76336}$,

where $T_{\mathrm{S}}$ is the scan duration and $X_{\mathrm{zpd}}$ is the ZPD position in the vicinity of the median of 1-76336. 


\section{TANSO-FTS on orbit characterization and calibrations}

The overall radiometric and spectroscopic performances are good and they are well within design objectives. Figure 6 shows the measured $\mathrm{CO}_{2}$ spectrum of the Level $1 \mathrm{~B}$ V050.050 along with the modeled spectrum for the retrieval V01.10 and the residual difference (Yoshida et al., 2011). The residual is close to the noise level and the spectral resolution is as designed. In this section, the calibrations after launch are described. The instrument model and the items, which need corrections, are summarized in Tables 2 and 3, respectively. The calibration events in chronological order were described in Kuze et al. (2011b). The timedependent degradation of the TANSO-FTS throughput (response) can be cross-checked by the methods described in the following subsections.

\subsection{Radiometric calibration and on-orbit degradation}

There are several ways for TANSO-FTS to estimate the calibration accuracy and degradation on orbit. SWIR and TIR are calibrated independently.

\subsubsection{Prelaunch calibration}

As described in detail in Kuze et al. (2009) and Sakuma et al. (2010), the TANSO radiometric response was calibrated before launch by using integrating spheres. An example of the radiance conversion factor is shown in Fig. 7. TANSOFTS has channeling in Band $2 \mathrm{~S}$, which is caused by multiple reflections at the band separation optics, and its magnitude is stable. As demonstrated in Fig. 7, a small oscillation feature of the conversion factor corrects channeling simultaneously and produces channeling-corrected spectral radiance. To correct atmospheric absorption in the laboratory and draw the envelope, we used the thermal vacuum chamber test results that are not affected by absorption. An absolute radiometric accuracy of better than $\pm 5 \%$ was achieved prior to launch in order to achieve better than $1 \% X_{\mathrm{CO}_{2}}$ accuracy.

\subsubsection{Vicarious calibration for SWIR}

The first vicarious calibration was performed in the summer of 2009. The measurement campaign was conducted using a dry lakebed in Railroad Valley (RRV), Nevada, USA with the NASA Atmospheric $\mathrm{CO}_{2}$ Observations from Space (ACOS) team (Kuze et al., 2011a). The spectral radiance was calibrated by comparing the satellite-measured radiances and simulated radiances at the top of the atmosphere (TOA). The observations at RRV suggest that the responsivities have changed by $-9 \pm 7 \%,-1 \pm 7 \%$ and $-4 \pm 7 \%$ for bands 1,2 , and 3 , respectively, from the values corresponding to prelaunch calibration. In 2010 and 2011, the second and third campaigns were performed and the accuracy was improved by more frequent calibration of the sur-

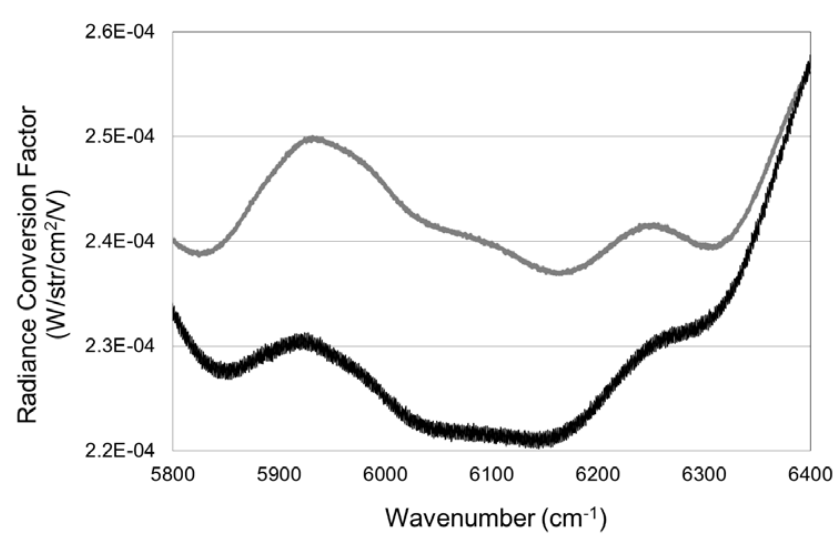

Fig. 7. Radiance conversion factors from the Level $1 \mathrm{~B}$ data to the spectral radiance for the TANSO-FTS Band $2 \mathrm{P}$ (bold gray line) and $2 \mathrm{~S}$ (solid line) of high gain.

face albedo measurements (Kuze et al., 2011d, 2012). As shown in Table 4a, the changes became slower 18 months after launch. The Airborne Visible/InfraRed Imaging Spectrometer (AVIRIS) on ER-2 aircraft had flown over RRV twice at the time of GOSAT overpass on 9 October 2009 and 20 June 2011. Cross-calibrations between AVIRIS and GOSAT independently corroborated the vicarious calibration results and showed a similar trend of the degradation. Further analysis is needed for AVIRIS data.

\subsubsection{Solar calibration for SWIR}

The back side of the Spectralon diffuser plate is exposed for a short time once a month. If the degradation is proportional to the exposure time, it is safe to assume that the back side has not been degraded. Therefore, the back side calibration data permit monitoring of the relative degradation. The solar irradiance calibration measurements have been analyzed for three years. On 4 March 2009, by rotating the whole satellite itself, the incident angle dependence of the onboard Spectralon reflectivity was acquired. We used this data and applied linear interpolation for cosine effect correction instead of simply normalizing with a cosine function. For this analysis, the Sun and Earth seasonal variation in distance was also corrected. Figure 8a shows the ratio between the front and back sides of the solar diffuser plate calibrations. The figure shows a large degradation in TANSO-FTS Band 1 on the diffuser's front surface, but slowing down of the degradation with time. Figure $8 \mathrm{~b}$ shows monthly solar calibration data using the diffuser plate's back side. The curve indicates the on-orbit radiometric response change of the instrument itself. In the first year after launch, the degradation of both the diffuser and the TANSO-FTS throughput was fast. However, the degradation became considerably slower in the following year. The three vertical lines in each panel of Fig. 8 represent the times of the vicarious calibration 
Table 2. TANSO-FTS and CAI models.

\begin{tabular}{|c|c|c|c|}
\hline & & Number of models & Notes (conversion and relations) \\
\hline \multirow[t]{2}{*}{ Radiometric } & FTS & 3 bands by 2 linear polarizations and by 2 gains & $\begin{array}{l}\text { From TANSO-FTS Level 1B Spectra in V } \mathrm{cm} \\
\text { to spectral radiance }\end{array}$ \\
\hline & CAI & 4 bands, dark current model & $\begin{array}{l}\text { From TANSO-CAI Level } 1 \mathrm{~A} \text { data and } \\
\text { integration time to spectral radiance }\end{array}$ \\
\hline \multirow{2}{*}{ Spectroscopic } & FTS & 4 bands (16 ILSFs) & FTS ILSF for convolution \\
\hline & CAI & 4 bands & CAI spectral response \\
\hline \multirow{2}{*}{ Geometric } & FTS & - & From pointing mirror angle to viewing vector \\
\hline & CAI & 4 bands (AT and CT) & From pixel number to viewing vector \\
\hline Polarization & FTS & 3 bands (Mueller matrix) & P, S output and 2 Stokes parameters \\
\hline
\end{tabular}

Table 3. TANSO-FTS and CAI correction factors.

\begin{tabular}{|c|c|c|c|}
\hline & & Number of models & Functions \\
\hline \multirow{2}{*}{ Radiometric } & FTS & 3 bands by 2 Linear polarizations & Days from launch \\
\hline & CAI & 4 bands & Days from launch \\
\hline Spectroscopic & FTS & Common value for all 4 bands & Days from launch \\
\hline Geometric & FTS & - & Combination of AT and CT angels \\
\hline
\end{tabular}

campaigns. The degradation between June 2009, 2010, and 2011 is summarized in Table $4 b$ and c.

\subsubsection{SWIR trend over the Sahara desert}

The Sahara and Arabian deserts are virtually clear all year round with almost no vegetation and water. We picked several sites as shown in Fig. 9a. The surface is not always Lambertian and the reflectance is dependent on the solar zenith angle. To estimate the degradation accurately, data from the same time of the year have to be compared. To minimize the random noise, several data in the same season are selected and averaged. Since August, 2010, we have used the 3-point cross-track scan mode as the nominal one. Earlier measurements were made primarily in the 5-point cross-track scan mode. We compared June and July of 2009 and 2010 data using the 5-point cross-track scan mode and 2011 data using the 3-point cross-track scan mode. TANSOFTS TIR data between $898 \mathrm{~cm}^{-1}$ and $929 \mathrm{~cm}^{-1}$ was used for cloud screening. If the maximum brightness temperature is lower than $292.5 \mathrm{~K}$, we then assume that the data is cloud-contaminated. This screening process is applied only for the Sahara desert trend analysis. We recorded the spectral radiances of $12971.027 \mathrm{~cm}^{-1}, 6008.327 \mathrm{~cm}^{-1}$, and $4806.9806 \mathrm{~cm}^{-1}$ in the analysis for bands 1,2 , and 3. Figure $9 \mathrm{~b}$ shows the degradation of the TANSO-FTS throughput for the 5 sites in one year. The change in one year was derived from a weighted average of the data over the sites, respectively, on the basis of the assumption that the aerosol optical thicknesses are the same. Since we cannot compare Sahara 8 and 9 sites, shown in Fig. 9a from the 3-point crosstrack scan mode, we compared the data for June 2009, 2010 and 2011 at 3 sites instead. The results are summarized in Table $4 \mathrm{~b}$ and $\mathrm{c}$.

\subsubsection{SWIR degradation estimation and correction}

Even though $X_{\mathrm{CO}_{2}}$ and $X_{\mathrm{CH}_{4}}$ are retrieved by using differential absorption spectra, scattering by aerosol and thin clouds in the real atmosphere is still not negligible. To distinguish the reflection from the surface and scattering from the atmosphere for accurate light path estimation, the throughput degradation has to be monitored in order to maintain the radiometric accuracy in the entire mission. Table 4 summarizes the degradation derived from independent methods. From the vicarious calibration (absolute), onboard calibration using the solar diffuser plate (relative) and the data obtained for the analysis over the Sahara desert (relative), the analytical results are consistent. Agreement among the three independent methods for the estimation of one year degradation is within $3 \%$ (peak to peak). All methods indicate the radiometric response degradation with time in both TANSOFTS and CAI. Two types of degradation are observed. The first is a step change between the prelaunch value and the on-orbit initial value, especially in TANSO-FTS Band 1 and CAI bands 1 and 4. The calibration on TANSO-CAI is to be discussed in the next section. The second type of degradation is a gradual on-orbit change. The largest degradation occurs at the shortest wavelengths. There are a few possible explanations for this step change: (i) contamination during launch or subsequent deployment; (ii) change in the modulation efficiency of the TANSO-FTS mechanism, which is sensitive to the thermal and mechanical environmental conditions; and (iii) non-Lambertian angular distribution correction error in 
Table 4. Estimated degradations of radiometric responsivity for TANSO-FTS and CAI from four independent methods: (a) difference from prelaunch value and initial on-orbit calibration: degradation in one year (relative): (b) between June 2009 and June 2010 and (c) between June 2010 and June 2011. Sahara Desert includes July data.

(a)

\begin{tabular}{|c|c|c|c|c|c|c|c|c|}
\hline & & \multicolumn{3}{|c|}{ TANSO-FTS } & \multicolumn{4}{|c|}{ TANSO-CAI } \\
\hline & & B1 & B2 & B3 & B1 & $\mathrm{B} 2$ & B3 & B4 \\
\hline \multirow{3}{*}{ Vicarious calibration } & June 2009 & $-11 \%$ & $-1 \%$ & $-3 \%$ & $-17 \%$ & $+4 \%$ & $0 \%$ & $-18 \%$ \\
\hline & June 2010 & $-14 \%$ & $-2 \%$ & $-5 \%$ & $-20 \%$ & $-3 \%$ & $-4 \%$ & $-19 \%$ \\
\hline & June 2011 & $-14 \%$ & $-4 \%$ & $-6 \%$ & $-22 \%$ & $-7 \%$ & $-6 \%$ & $-20 \%$ \\
\hline
\end{tabular}

(b)

\begin{tabular}{|c|c|c|c|c|c|c|c|}
\hline \multirow{2}{*}{ Between June 2009 and June 2010} & \multicolumn{3}{|c|}{ TANSO-FTS } & \multicolumn{4}{|c|}{ TANSO-CAI } \\
\hline & B1 & B2 & B3 & B1 & B2 & B3 & B4 \\
\hline Vicarious calibration & $-3 \%$ & $-1 \%$ & $-2 \%$ & $-3 \%$ & $-7 \%$ & $-4 \%$ & $-1 \%$ \\
\hline Solar diffuser plate & $-2.4 \%$ & $-0.7 \%$ & $0.0 \%$ & N/A & N/A & N/A & N/A \\
\hline Sahara Desert & $-2.0 \%$ & $+0.2 \%$ & $-0.2 \%$ & $-1.7 \%$ & $-3.9 \%$ & $-0.7 \%$ & $-0.3 \%$ \\
\hline
\end{tabular}

Average of bands $\mathrm{P}$ and $\mathrm{S}$

(c)

\begin{tabular}{lrrrrrrrr}
\hline \multirow{2}{*}{ Between June 2010 and June 2011 } & \multicolumn{3}{c}{ TANSO-FTS } & & \multicolumn{3}{c}{ TANSO-CAI } \\
\cline { 2 - 5 } \cline { 5 - 7 } & B1 & B2 & B3 & & B1 & B2 & B3 & B4 \\
\hline Vicarious calibration & $0 \%$ & $-2 \%$ & $-1 \%$ & & $-2 \%$ & $-4 \%$ & $-2 \%$ & $-1 \%$ \\
Solar diffuser plate & $-0.6 \%$ & $-0.2 \%$ & $+0.1 \%$ & & N/A & N/A & N/A & N/A \\
Sahara desert & $-0.9 \%$ & $-0.5 \%$ & $-0.2 \%$ & & $+0.2 \%$ & $-1.3 \%$ & $-0.4 \%$ & $-0.6 \%$ \\
\hline
\end{tabular}

Average of bands $\mathrm{P}$ and $\mathrm{S}$.

the integrating sphere used for the prelaunch calibration. The gradual degradation may be attributed to contamination or changes in modulation efficiency. The upper part of Fig. 1 shows the schematics of the TANSO-FTS optics module. CAM is mounted between the pointing mirror and FTSmechanism. CAM data over the Sahara has shown no significant degradation since launch. The possible degradation may occur in modulation efficiency of the FTS mechanism or the aft optics optical efficiency.

Lunar calibration has been performed twice a year since launch, on 11 March and 9 April 2009, 28 April and 6 June 2010, and 18 April and 14 July 2011. The bidirectional reflectance distribution function (BRDF) for the lunar surface is not Lambertian and a complicated calibration is needed for absolute calibration. These data can be used for both TANSO-FTS and CAI absolute radiometric calibration. However, the lunar surface has a strong BRDF when the incident angle is zero. After the BRDF correction, the analytical results of TANSO-CAI show a similar trend, but further investigations and BRDF corrections are needed. In case of TANSO-FTS, the size of the moon disk viewed from the GOSAT orbit is about half of the instantaneous field of view (IFOV) making the analysis more complicated.
On the basis of the vicarious absolute calibration, response correction factors have been derived as a function of time (days from launch). By combining the prelaunch radiance conversion factor and these correction factors, the calibrated spectral radiance thus can be calculated. Because the surface reflectance over RRV and the solar diffuser reflectivity are high, the interferograms are calibrated with the medium gain to avoid saturation. High gain response over the Australian desert can be compared by observing the same site with high and medium gains. The ratio between the high and medium gains is determined by the feedback resistance of the amplifier. The comparison over the Australian desert indicated that the ratio is as designed. Thus, response correction factors derived from the medium gain can be applied to the high gain data. Low gain has never been used since launch.

\subsubsection{Cross-calibration and validation for TIR}

TIR spectral radiance is calibrated frequently by looking at DS and the onboard BB. Onboard calibration accuracy does not change with time unless the temperature sensor degrades. In parallel, cross-calibration with other space-borne instruments has been performed. The spectral radiance has been compared at high latitude by using simultaneous nadir 

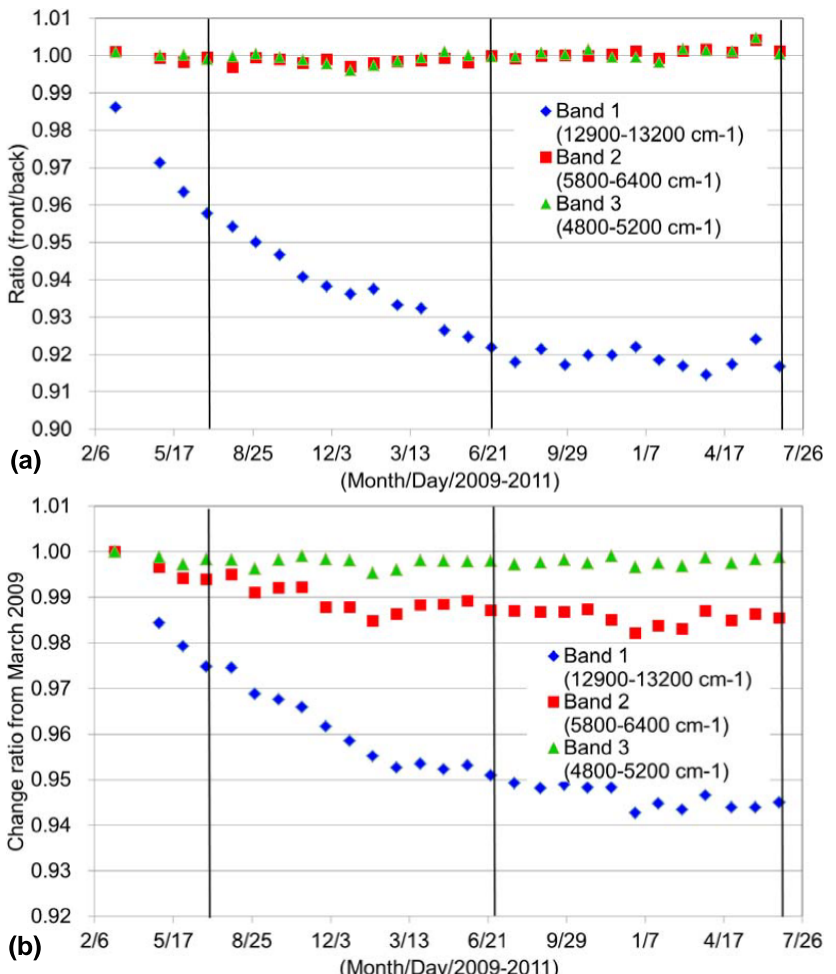

Fig. 8. Solar irradiance monthly calibration data from the onboard Spectralon diffuser, which indicates the change from the first measurement in space after correction for the distance between the satellite and the Sun and the angle of incidence of the solar beam upon the diffuser. The vertical lines represent the time of the 2009, 2010 and 2011 vicarious campaigns: (a) ratio of the front side radiance to the back side and (b) back side calibration.

overpasses of Infrared Atmospheric Sounding Interferometer (IASI). IASI data can be found within $100 \mathrm{~km}$ of the GOSAT field of view and within $10^{\circ}$ of nadir. The difference in brightness temperature between $200 \mathrm{~K}$ and $270 \mathrm{~K}$ is smaller than $\pm 1 \mathrm{~K}$ (Knuteson, private communication, 28 February 2011). In addition, TIR vicarious calibration was performed at RRV in June 2011 by measuring surface temperature and emissivity by Atmospheric Emitted Radiance Interferometer (AERI) FTS together with upward spectral radiation by using the airborne Scanning High-resolution Interferometer Sounder (S-HIS) FTS (Tobin et al., 2006; Kuze et al., 2011d). For an atmospheric window region around $10 \mu \mathrm{m}$, where the radiation from the surface is dominant, it is very difficult for IASI and GOSAT to view exactly the same location at the same time. The S-HIS data covered the entire IFOV of the TANSO-FTS and the target surface temperature of RRV is $320 \mathrm{~K}$. However, we cannot compare the radiation from the atmosphere above the S-HIS flight altitude. Only satellite data can provide the thermal radiation spectra from the upper atmosphere where the radiation from the surface is fully absorbed. A combination of formation flight of a highaltitude $(20 \mathrm{~km})$ aircraft and match-up data from other satel-

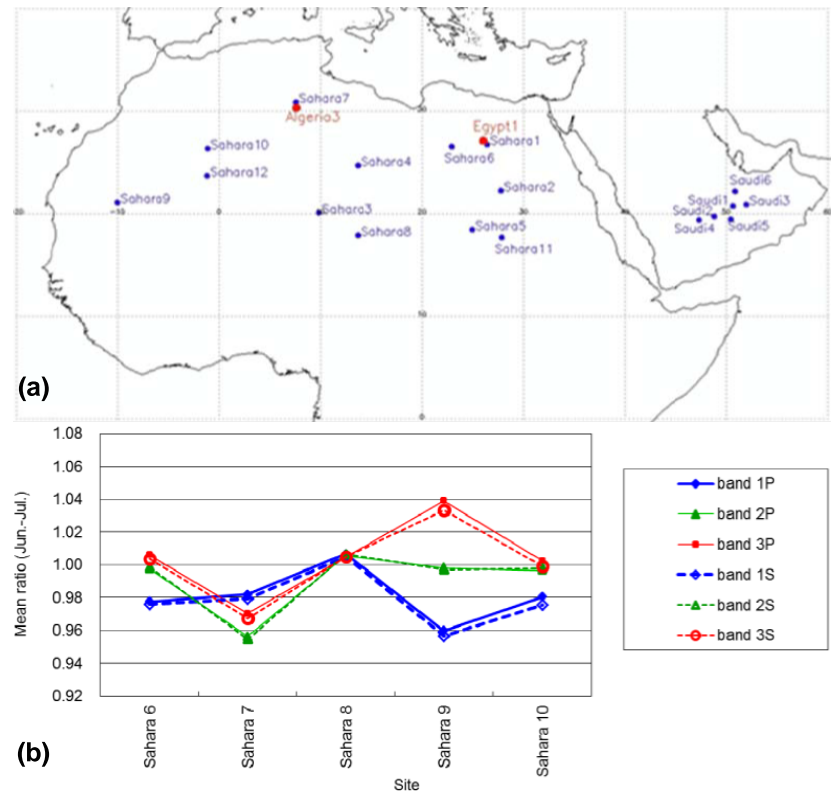

Fig. 9. (a) Radiance monitoring sites over the Sahara and Arabian deserts. (b) TANSO-FTS degradation in one year between 2009 and 2010 over the Sahara desert.

lites can calibrate the wide range of spectral radiance from both atmosphere and ground surface. Both cross-calibrations with IASI and S-HIS agree well within $\pm 1 \mathrm{~K}$. These data are still being analyzed and compared.

\subsection{Signal-to-noise ratio}

There is no stable onboard white light source available for radiometric calibration purposes to estimate the noise level along with a known signal level. Therefore, SNR cannot be measured explicitly. It is estimated from the imaginary spectra or out-of-band real spectra. Currently the noise level is the same as measured before launch. There is no significant response degradation. Thus, we can expect the SNR performance to be reasonably similar to that described by Kuze et al. (2009). There are two dominant noise sources for TANSO-FTS bands 1, 2, and 3. One is the detector and its electronics noise, which is independent of the input signal level and proportional to the square root of the electric band width. The performance can be measured with dark input data and can be expressed with specific detectivity $\left(D^{*}\right)$. The second source is the shot noise, which is proportional to the square root of the total number of input photons. Band 2 has wide optical band width of $5800-6400 \mathrm{~cm}^{-1}$ to cover $\mathrm{CO}_{2}, \mathrm{CH}_{4}$ and $\mathrm{H}_{2} \mathrm{O}$ lines, here the shot noise contribution is larger than in the other two bands. 


\subsection{Spectral resolution and instrument line shape function}

\subsubsection{Prelaunch calibration and simulated model}

The geophysical parameters are retrieved by comparing the measured spectral radiance and the simulated radiance at the top of the atmosphere. $X_{\mathrm{CO}_{2}}, X_{\mathrm{CH}_{4}}$, and other geophysical parameters such as surface pressure can be retrieved by minimizing the residual between the measured spectrum and simulated spectrum convolved with the instrument line shape function (ILSF). Because ILSF is a function of the wavelength, ILSFs at selected wavelengths are modeled as shown in Fig. 10a. The models are simulated because the prelaunch tests with monochromatic light from tunable diode lasers were performed at limited wavenumbers and did not cover the whole spectral range. Off-axis mounting of the detector due to imperfect alignment creates an asymmetry of ILSF; however, this effect can be numerically simulated. The ILSF at short wavelengths is more sensitive to the misalignment, thus the detector position during the integration has to be accurately measured. Misalignment from the ideal optical axis of the Band 1P and 1S are estimated by introducing monochromatic light from tunable diode lasers prior to launch. Spatial uniformity over the scene aperture and angular uniformity over the entire IFOV are particularly important for this characterization. Although the prelaunch test configuration was imperfect, the data nevertheless contains detector offset information. By carefully simulating the imperfect prelaunch test configuration, the offset value was estimated. The best estimate ILSF models were calculated using the offset value and optical aberration. On the other hand, for bands 2,3 , and 4, ILSF are much less sensitive to the misalignment of the detector, and being less than $50 \mu \mathrm{m}$, the finite size effect of IFOV has been considered.

\subsubsection{Spectral resolution on orbit}

Figure $10 \mathrm{~b}$ shows the long term stability of the data using the onboard ILSF calibration laser which has $6460 \mathrm{~cm}^{-1}$ frequency. Although the frequency of the onboard diode laser without temperature control is not constant and the laser cannot illuminate the solar diffuser plate uniformly, the ILSF shape itself has been very stable since launch; this indicates that no significant spectral resolution change has been observed.

\subsubsection{Absolute spectral calibration}

By carefully monitoring sampling laser output level of the FTS mechanism and the solar Fraunhofer line positions, we have observed a gradual decrease of the laser signal detection level and an increase of the apparent wavenumber of the Fraunhofer lines. The degradation of laser signal detection level is exponential and smaller than $10 \%$ in one year and is slowing down. We estimate that the laser will still have a
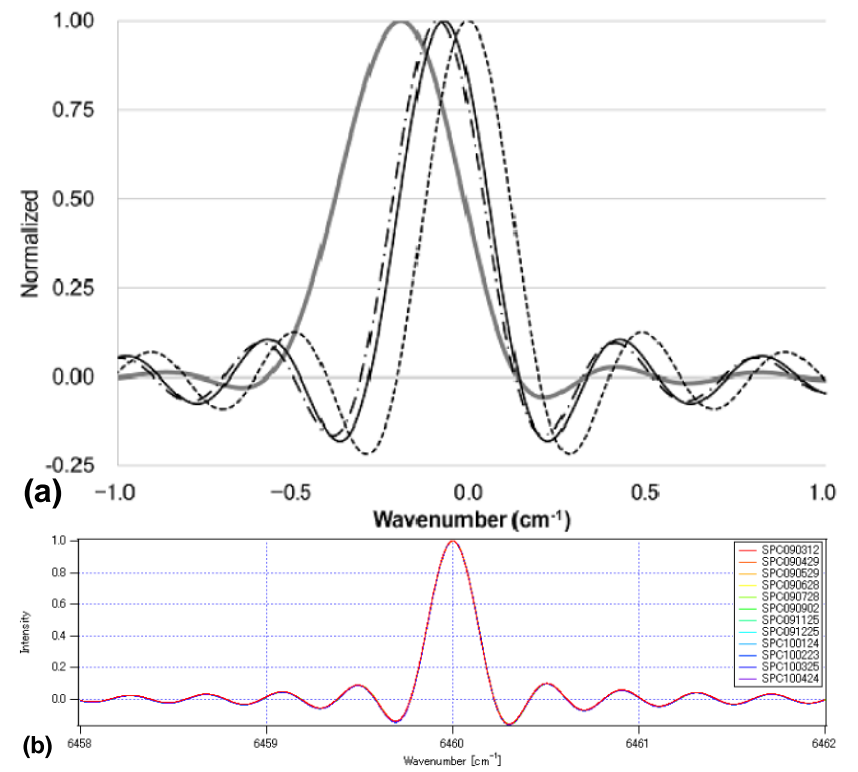

Fig. 10. (a) ILSF models at typical wavelength of each spectral band: $13050 \mathrm{~cm}^{-1}$ of Band 1P (bold gray), $6025 \mathrm{~cm}-1$ of Band 2 (dash-dotted), $5000 \mathrm{~cm}^{-1}$ of Band 3 (solid) and $700 \mathrm{~cm}^{-1}$ of Band 4 (dotted). (b) Overwritten ILSF acquired monthly on orbit from March 2009 to April 2010 using the onboard diode laser which has $6460 \mathrm{~cm}^{-1}$ frequency.

sufficient level of control after 10 years of operation. We consider that the laser signal detection level decrease and the apparent increase in Fraunhofer wavenumbers are related. Because all bands have the same spectral calibration factors, the most probable cause is a change in optical alignment of the laser beam on orbit as illustrated in Fig. 11. As a consequence, the illumination on the laser detector has become weaker, maximum OPD has become larger, and spectral resolution has become slightly higher. The absolute spectral position can be calibrated by applying the spectral correction factor with time:

$v_{\text {corre }}=v_{\text {raw }} /\left(a_{0} \exp \left(a_{1} t\right)+a_{2}\right)$,

where $v_{\text {corre }}, v_{\text {raw }}$ are wavenumbers before and after the correction, and $a_{0}, a_{1}, a_{2}$, and $t$ are the calibration coefficients and time from launch. As shown in Fig. 12, the change since launch is less than $10 \mathrm{ppm}$ and the effect on spectral resolution is negligibly small.

\subsection{Geolocation and pointing characterization}

During interferogram scans, the pointing mirror is turned very slowly to perform IMC. Then, during turnarounds, it quickly steps to the next point of the grid pattern. This complicated control is sensitive to electromagnetic and microvibration environments. The angular resolvers of the two axis motors are the only devices to provide geometrical information for the onboard control as well as the data analysis on 


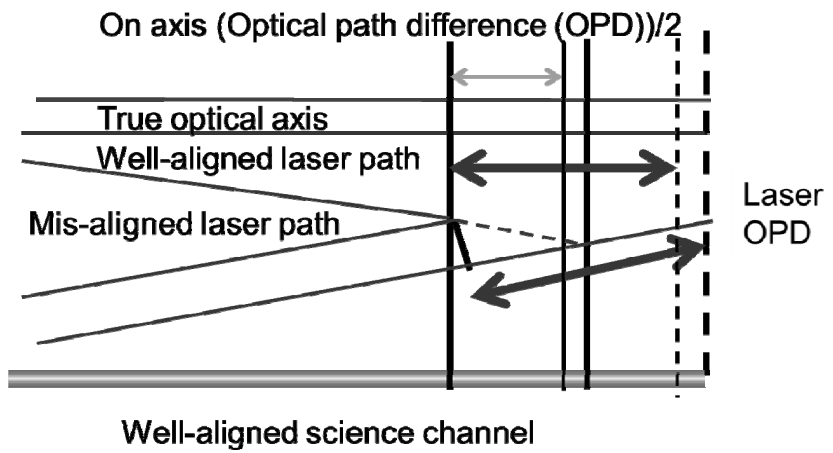

Fig. 11. Schematics of the sampling laser misalignment and OPD.

the ground. The resolvers are sensitive to their electromagnetic environment and are suspected to have errors, which are of two kinds. One kind of error is a known offset (predictable) that the onboard resolver can detect and that the Level 1B process can correct. The other is an unpredictable error, which the resolver cannot detect. The error is a possible resolver anomaly most likely due to noise induced by electromagnetic interference. The second error was first detected just three months after launch (April 2009) and had not been stable until August 2009. To estimate the offset values, we use images acquired with the Advanced Visible and Near Infrared Radiometer type 2 (AVNIR-2) on the Advanced Land Observing Satellite (ALOS) (Tadono et. al., 2009) and CAM data of the ground correction points (GCP). There is no general formula to express the pointing error. Instead we have been providing the offset information since September 2009 and an example is shown in Table 5. Offset values have slowly changed with time. It is a function of the scanning pattern and not of other considerations, such as the location on Earth. Regarding the pointing error, settling down (overshoot) is not ideal when the AT range of motion is large. The offset level is not a simple linear function of AT and CT angles. Hence, the geometrical correction in the 3-point crosstrack scan mode is not the same as that in the target mode and the 5-point cross-track scan mode.

\subsection{Polarimetric calibration by Mueller matrix}

Both instrument and scene flux scattered by the Earth's atmosphere have large polarization. The protection coating on the silver pointing mirror surface has a polarization phase. To calculate the response to the polarized input scene flux, the polarization reflectivity and phase of the instrument need to be considered. The relation between input flux Stokes vector and two linear polarizations " $\mathrm{P}$ " and " $\mathrm{S}$ " of TANSO-FTS measurements can be expressed using the Mueller matrix as follows:

$$
\begin{aligned}
S_{P_{\text {output }}=} & \mathbf{M}_{\mathrm{pp}} \mathbf{M}_{\mathrm{opt}} \mathbf{M}_{\mathrm{r}}\left(-\theta_{\mathrm{CT}}\right) \mathbf{M}_{\mathrm{p}}\left(\theta_{\mathrm{AT}}\right) \mathbf{M}_{\mathrm{m}}\left(\theta_{\mathrm{AT}}\right) \\
& \mathbf{M}_{\mathrm{r}}\left(\theta_{\mathrm{CT}}\right) S_{\text {input }},
\end{aligned}
$$

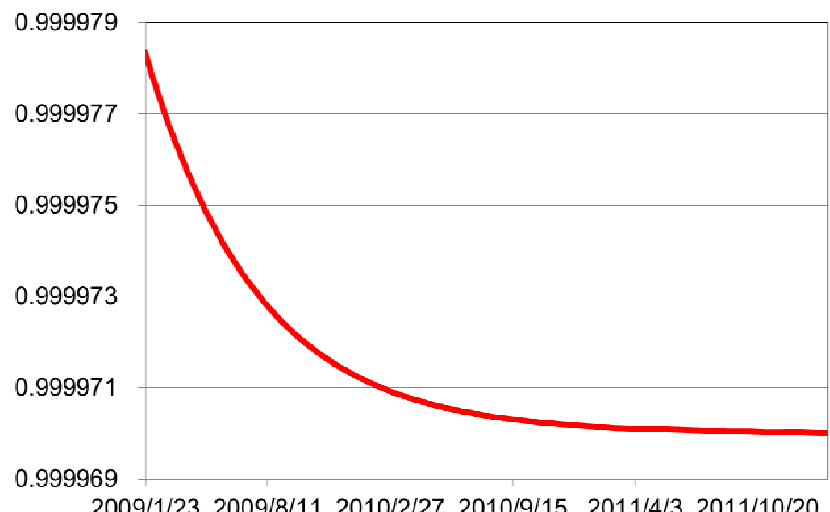

2009/1/23 2009/8/11 2010/2/27 2010/9/15 2011/4/3 2011/10/20

Fig. 12. Spectral calibration factor as a function of days from launch.

$$
\begin{aligned}
S_{S_{\text {ouput }}=} & \mathbf{M}_{\mathrm{ps}} \mathbf{M}_{\mathrm{opt}} \mathbf{M}_{\mathrm{r}}\left(-\theta_{\mathrm{CT}}\right) \mathbf{M}_{\mathrm{p}}\left(\theta_{\mathrm{AT}}\right) \\
& \mathbf{M}_{\mathrm{m}}\left(\theta_{\mathrm{AT}}\right) \mathbf{M}_{\mathrm{r}}\left(\theta_{\mathrm{CT}}\right) S_{\text {input }},
\end{aligned}
$$

where $\mathbf{M}_{\mathrm{pp}}, \mathbf{M}_{\mathrm{ps}}, \mathbf{M}_{\mathrm{opt}}, \mathbf{M}_{\mathrm{p}}, \mathbf{M}_{\mathrm{m}}$, and $\mathbf{M}_{\mathrm{r}}$ are Mueller matrixes of polarization beam splitter, optical efficiency of the FTS-mechanism and aft optics, phase difference due to the pointing mirror surface coating, pointing mirror reflectivity, and CT rotation, respectively. $S_{P_{\text {output }}}, S_{S_{\text {output }}}, S_{\text {input }}$, $\theta_{\mathrm{CT}}$, and $\theta_{\mathrm{AT}}$ are output and input signals of the Stokes vector and the CT and AT angles, respectively. $S_{\text {input }}$, the Stokes vector at the top of the atmosphere, can be calculated with a vector radiative transfer model. By comparing the simulated $I$ components of the Stokes vector $S_{P_{\text {output }}}$ and $S_{S_{\text {output }}}\left(I_{\text {PopC }}\right.$ and $\left.I_{\text {SopC }}\right)$ and $I_{\text {PopM }}$ and $I_{\text {SopM }}$ measured spectral radiance with TANSO-FTS, $X_{\mathrm{CO}_{2}}$ and $X_{\mathrm{CH}_{4}}$ can be independently retrieved from SWIR P and S bands. The reference plane for polarization for the input Stokes vector, of which components are $I, Q, U$, and $V$, is defined by the local normal at the target and the ray from the target to the satellite. The prelaunch radiometric calibration was performed on the ground by introducing un-polarized light from nadir, of which input and output spectral radiance are $I_{\mathrm{ipPL}}, I_{\mathrm{PopPL}}$ and $I_{\mathrm{SopPL}}$, respectively. The prelaunch calibration data with the Level 1 format can be expressed in $R_{\mathrm{P}}\left(I_{\mathrm{PopPL}}\right)$ and $R_{\mathrm{S}}\left(I_{\mathrm{SopPL}}\right)$. The radiance conversion table prepared prelaunch shows the relation between $I_{\mathrm{ipPL}}$, $R_{\mathrm{P}}\left(I_{\mathrm{PopPL}}\right)$, and $R_{\mathrm{S}}\left(I_{\mathrm{SopPL}}\right)$ as shown in Fig. 7. $I_{\mathrm{PopPL}}$ and $I_{\text {SopPL }}$ are not used explicitly in radiometric calibration.

The above mentioned Mueller matrix needs to be used to correct the AT and CT angle dependency of the pointing mirror in the case of slant looking. Thus the correction requires the pointing mirror reflectivity and phase information in $\mathbf{M}_{\mathrm{p}}$ and $\mathbf{M}_{\mathrm{m}}$ as the function of AT angle (the incident angle to the mirror) as indicated in Figs. 13 and 14a. These data are acquired with the mirror witness sample, which was coated at the same time as the flight mirrors. Aft optics are calibrated prelaunch as the overall response, including the pointing mirror, fold mirror, FTS mechanism, and telescope; the 
Table 5. TANSO-FTS pointing onboard anomaly and correction: upper table is for the 5-point cross-track mode scan and lower one is for the 3-point cross-track scan mode. IDs in the tables are illustrated in Fig. 2. These are typical values and the offsets have changed with time.

\begin{tabular}{rrlrl}
\hline \multicolumn{6}{l}{ (a) 5-point cross-track scan mode } \\
ID & AT $(\mathrm{km})$ & Direction & CT $(\mathrm{km})$ & Direction \\
\hline 0 & 8.3 & positive down track & 2.1 & the left of the ground track ** \\
1 & -0.5 & negative down track & -0.4 & \\
2 & 0.0 & positive down track & -0.6 & \\
3 & -0.7 & & -0.6 & the right of the ground track \\
4 & -0.1 & negative down track & -0.4 & \\
5 & 9.2 & & & \\
6 & 0.7 & positive down track & -0.1 & the left of the ground track \\
7 & -0.3 & & -0.3 & \\
8 & -1.1 & negative down track & -0.4 & the right of the ground track \\
9 & -1.3 & & -0.5 & \\
\hline
\end{tabular}

(b) 3-point cross-track scan mode (typical values)

\begin{tabular}{|c|c|c|c|c|}
\hline ID & AT (km) & Direction & $\mathrm{CT}(\mathrm{km})$ & Direction \\
\hline 0 & 5.0 & \multirow{18}{*}{ positive down track } & 3.0 & \multirow{18}{*}{ the left of the ground track } \\
\hline 1 & 5.1 & & 3.0 & \\
\hline 2 & 5.4 & & 3.2 & \\
\hline 3 & 5.2 & & 2.6 & \\
\hline 4 & 5.2 & & 2.8 & \\
\hline 5 & 5.2 & & 2.8 & \\
\hline 6 & 5.7 & & 3.5 & \\
\hline 7 & 6.1 & & 3.7 & \\
\hline 8 & 5.8 & & 3.6 & \\
\hline 9 & 6.0 & & 3.0 & \\
\hline 10 & 5.9 & & 3.0 & \\
\hline 11 & 5.8 & & 3.0 & \\
\hline 12 & 6.2 & & 2.9 & \\
\hline 13 & 6.2 & & 3.0 & \\
\hline 14 & 6.2 & & 3.0 & \\
\hline 15 & 5.0 & & 3.5 & \\
\hline 16 & 5.0 & & 3.6 & \\
\hline 17 & 5.6 & & 3.7 & \\
\hline
\end{tabular}

* Forward looking = looking south-southwest at the equator for the descending track (dayside). ** Left of the ground track $=$ the east-southeast at the Equator for the descending track (dayside).

individual optical efficiency is not needed for the radiometric calibration for the on-orbit data. This coating has been optimized for the SWIR region with higher than $99 \%$ reflectivity and the AT angle dependency of SWIR bands is very small.

However, the reflectivity is lower and the polarization sensitivity is higher in some TIR wavelength, as shown in Fig. 14b. As discussed in Sect. 2.6, measured spectral radiance $S_{T_{\text {output }}}$ can be expressed using the scene flux $S_{T_{\text {input }}}$, thermal radiation from the pointing mirror, and the background radiation in the following Mueller matrix:

$S_{T_{\text {output }}}=\mathbf{M}_{\text {opt }} \mathbf{M}_{\mathrm{r}}\left(-\theta_{\mathrm{CT}}\right) \mathbf{M}_{\mathrm{m}}\left(\theta_{\mathrm{AT}}\right) \mathbf{M}_{\mathrm{r}}\left(\theta_{\mathrm{CT}}\right) S_{\text {Tinput }}$

$+\mathbf{M}_{\mathrm{opt}} \mathbf{M}_{\mathrm{r}}\left(-\theta_{\mathrm{CT}}\right) \mathbf{M}_{\mathrm{e}}\left(\theta_{\mathrm{AT}}\right) S_{\mathrm{Tmirror}}+\Delta S_{\mathrm{BG}}$,

where $\mathbf{M}_{\mathrm{e}}, S_{T_{\text {mirror }}}$, and $\Delta S_{\mathrm{BG}}$ are Mueller matrix of pointing mirror emissivity, Stokes vectors of the radiation from the pointing mirror, and the background radiation variation between calibrations, respectively. $I$ component of $S_{T_{\text {input }}}$ is retrieved from Eq. (11) in the TIR Level 1 processing.

\subsection{Quality flag of data products}

All the data transmitted to the ground is processed to the Levels $1 \mathrm{~A}$ and $1 \mathrm{~B}$ products and is known to contain some reduced quality data. In the Level 1 processing, quality is checked and data with problems can be clearly identified with flags, as listed in Table 6 . The noise-spike quality flag in the Level 1B product can be triggered by rapid fluctuation of the interferogram intensity and by spikes caused by cosmic rays. For the three years since launch, no spikes caused by cosmic rays have been detected. FTS mechanism scan speed instabilities larger than $2 \%$ would be detected by monitoring the passing time of 10 different OPD positions. However, the 

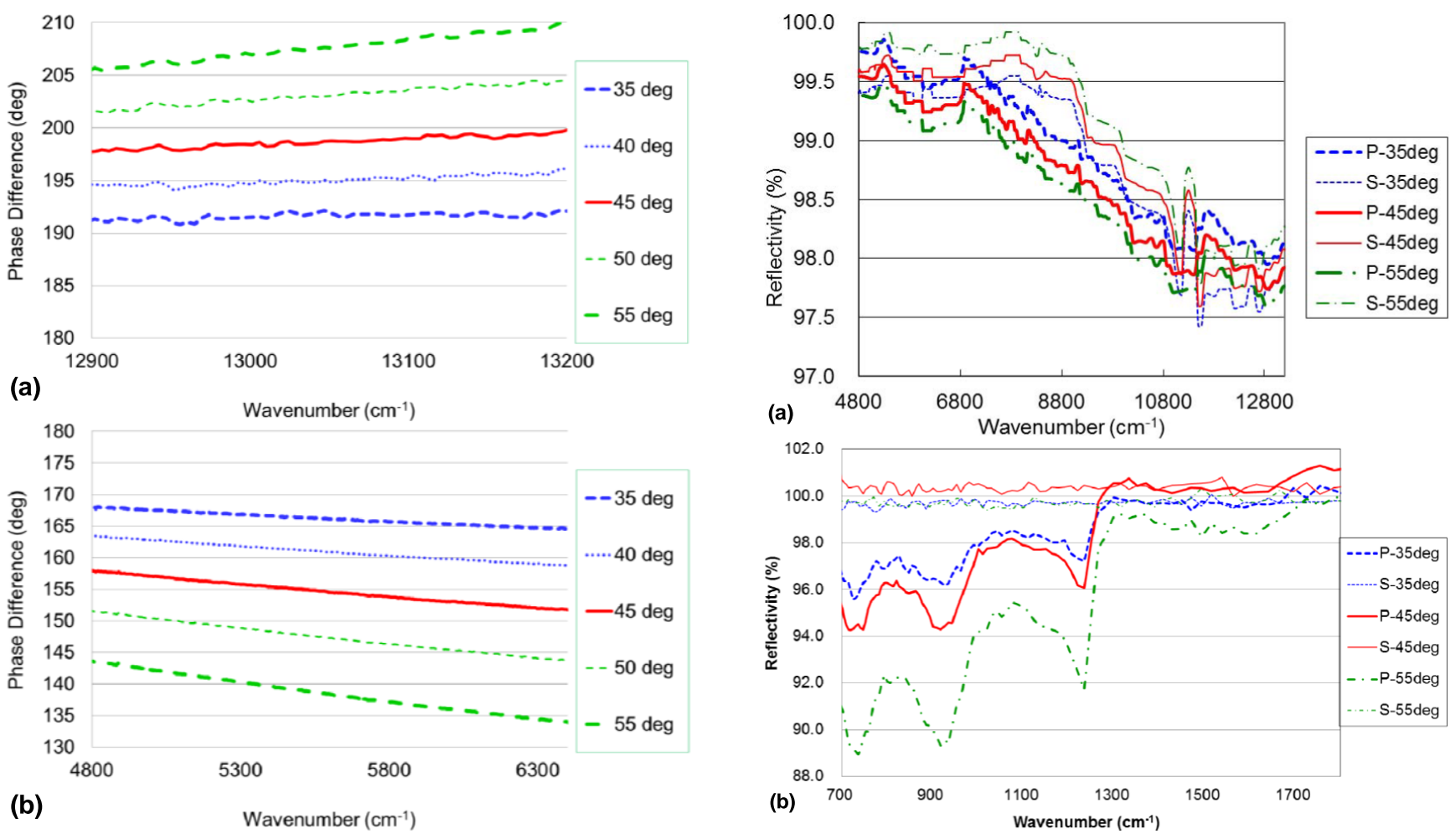

Fig. 13. Measured phase of the pointing mirror using the witness sample for Mueller matrix of incident angles of 35, 40, 45, 50 and 55 deg: (a) Band 1 and (b) bands 2 and 3.

stability on orbit has so far been much better than $1 \%$ and so the speed-instability flag has not yet been triggered.

\subsection{Additional corrections}

Methods of conversion, calibration and correction are described in this paper. The Level 1 products and calibration data include all the information required to produce Level 2 products. In parallel, for the detailed Level 1 product analysis, additional software corrections are applied as follows:

(i) Finite size of the field of view; finite size of the field of view has a self-apodization effect. Without large degradation of the spectral shape, the self-apodization due to the size is corrected as described by Knuteson et al. (2004). Only Band 4 data can be additionally corrected for meteorological application.

(ii) Wavelength shift correction; the shift due to the optical alignment change of the sampling laser is corrected even though the value of the shift since launch is small enough.

(iii) Mueller matrix; four-by-four elements for 3 TANSOFTS SWIR bands are added to the Level 1 data.

Fig. 14. Measured spectral reflectivity of the pointing mirror using the witness sample for two linear polarizations ( $\mathrm{P}$ and $\mathrm{S})$ of the incident angles of 35, 45, and $55 \mathrm{deg}$ : (a) SWIR and (b) TIR.

(iv) Geometric correction; the most probable offset correction of the pointing mirror estimated from CAM data is applied.

(v) Spectral radiance conversion; the Level 1 data having units of $\mathrm{V} \mathrm{cm}$ are converted to the calibrated spectral radiance having units of $\mathrm{W} \mathrm{cm}{ }^{-1} \mathrm{str}^{-1}$.

\section{TANSO-CAI Level 1A data processing, characterization, and calibration}

The design and prelaunch performance and calibration of TANSO-CAI were described in detail in Kuze et al. (2009). The TANSO-CAI Level 1A data processing, on-orbit performance, and calibration are described in this section. Instrument models and calibrations are summarized in Tables 2 and 3. JAXA has been providing the raw Level 1 data to NIES and other users. In this section, we describe how to correct the raw data and convert it to spectral radiance.

\subsection{Radiometric calibration and correction}

All the pixels of 4 TANSO-CAI bands were calibrated using an integrating sphere (Kuze et al., 2009; Sakuma et al., 2010). Radiometric calibration is based on the prelaunch calibration and the data are corrected with the following processes. 
Table 6. Sampling interval non-uniformity as a function of OPD of the forward and backward scans.

\begin{tabular}{lll}
\hline Source & Item & Criteria \\
\hline Satellite & Orbit control & Data acquired during orbit control \\
\hline \multirow{2}{*}{ FTS } & Saturation & Interferogram saturation, Blackbody temperature \\
& Spike & Fluctuation, Cosmic ray \\
& Low frequency disturbance (jitter) & Satellite and point mechanism microvibration \\
& Pointing error & AT and CT pointing error larger than 0.1deg \\
& FTS mechanism temperature & Lower than $20^{\circ}$ or higher than $26^{\circ}$ \\
& Large ZPD position shift & Shift larger than 100 laser fringes \\
& Scan speed instability & FTS scan speed instability larger than $2 \%$ \\
\hline
\end{tabular}

\subsubsection{Offset subtraction and spectral radiance conversion}

The TANSO-CAI Level 1A data includes signals and offsets which vary depending on the total input radiance, and dark currents. The observed spectral radiance is calculated by extracting the offset and dark current using the following equation:

$S_{\text {obs }}(n)=\frac{V_{\mathrm{C}}(n)-V_{\mathrm{Cpre}}-V_{\mathrm{C} \text { dark }}\left(n, T_{\mathrm{int}}\right)}{T_{\mathrm{int}} R_{\mathrm{C}}(n)}$,

where $V_{\mathrm{C}}(n), V_{\text {Cdark }}(n)$, and $R_{\mathrm{C}}(n)$ are output, dark level, and response of the pixel number $\mathrm{n}$, respectively. $T_{\mathrm{int}}$ is the integration time of the detectors. $V_{\text {Cpre }}$ is the average of output of the first three prescan pixels and calculated separately for the odd and the even pixels. We assume that the dark level is a function of the integration time and is constant with time since launch. $R_{\mathrm{C}}(n)$ is based on the prelaunch calibration and degradation correction is described below.

\subsubsection{Pixel-to-pixel non-uniformity correction}

All the pixels of each band were calibrated at the same time before launch by using the integrating sphere, the inner surface of which is coated with barium sulfate $\left(\mathrm{BaSO}_{4}\right)$. Radiation emanating from the aperture of the integrating sphere has a non-Lambertian angular distribution, especially for bands 1 (UV) and 4 (SWIR). Spectral radiance of the integrating sphere was calibrated at a direction perpendicular to the aperture and its angular distribution was not calibrated before launch. As TANSO-CAI has a wide field of view, the prelaunch data other than the center pixel has to be corrected as follows. The telescope optics of bands 1 and 4 have very small vignetting while bands 2 and 3 have large vignetting. Therefore, the angular distribution of bands 1 and 4 can be corrected. To check pixel-to-pixel uniformity, we assume the averaged Earth albedo is uniform when TANSO-CAI data acquired from all the 44 paths over one year. The Earth albedo is defined as the measured reflectance at the top of the atmosphere. By correcting Rayleigh scattering and sun glint effect, pixel-to-pixel non-uniformity can be detected and corrected using the averaged measured data for Band 1. There

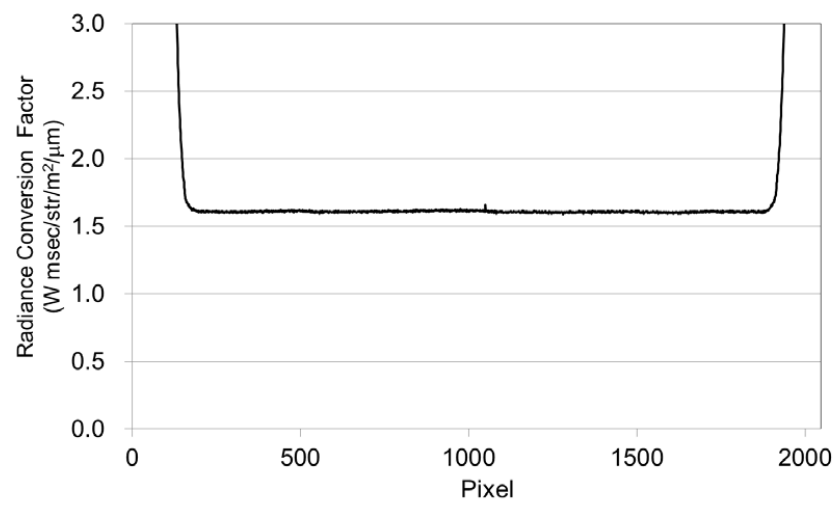

Fig. 15. Radiance conversion factors for the TANSO-CAI Band 1.

was no need for pixel-to-pixel correction for Band 2. For Band 3, local bump structures are corrected using one-year averaged data. Band 4 is corrected using one-year averaged data, except for several pixels, of which dark current have increased. Pixel 154 shows the sudden increase after passing through the South Atlantic Anomaly. The data shown in Fig. 15 is the pixel-to-pixel non-uniformity correction of Band 1 . These corrected radiance conversion factors are used for the TANSO-CAI Level 1B product.

\subsubsection{Degradation after launch}

The vicarious calibrations at RRV indicate that the difference between prelaunch and initial on-orbit response is large in TANSO-CAI bands 1 and 4 but that the subsequent response change with time on orbit seems to be slow for all bands. Another method to check the degradation is a simultaneous airplane flight with GOSAT (Kuze et al., 2011d). The preliminary comparison between AVIRIS and TANSO-CAI led to similar calibration results compared to the vicarious calibration. The last method is the comparison of the data over the Sahara desert as we did for TANSO-FTS. We had used the TANSO-CAI data between June of 2009, 2010 and 2011. First, we selected the site data where the measured Earth albedo was uniform to better than $0.5 \%$ across 9 ( 3 by 3) pixels of TANSO-CAI. Second, we used the sites where 


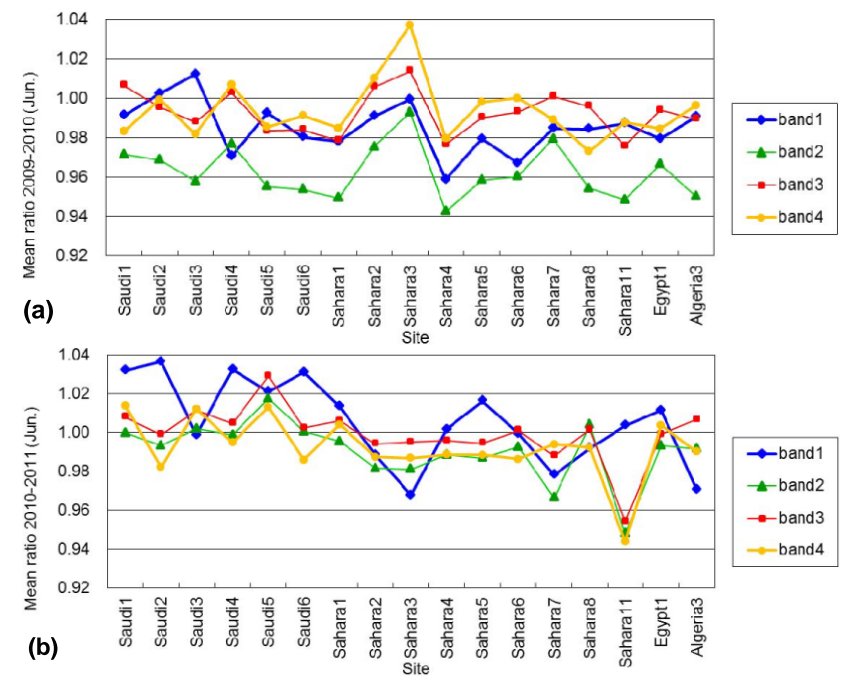

Fig. 16. TANSO-CAI degradation over the Sahara and Arabian deserts. (a) Between June 2009 and 2010, (b) between June 2010 and 2011.

normalized difference vegetation index (NDVI) is smaller than 0.01 . The one year degradation over each site is presented in Fig. 16. The variation of the measured Earth albedo differs from site to site and the weighted averages of response change over the 17 sites in one year of TANSO-CAI bands 1 , 2, 3, and 4 are summarized in Table $4 \mathrm{~b}$ and c. Lunar calibrations have been performed six times since launch. In addition to the difficulty to correct BRDF of lunar surface, the nonuniformity of the lunar surface reflectance has to be considered for TANSO-CAI. At present, lunar calibration data have not been used for radiometric degradation estimation. As illustrated in the lower part of Fig. 1, the TANSO-CAI optics have a simpler configuration than FTS and the response has become stable for all bands.

\subsection{Geolocation}

The exact viewing vector of each pixel was geometrically calibrated before launch with the collimator in the laboratory. As the optical aberration and misalignment of the off-axis pixels become larger, the geometrical model of both AT and CT directions can be well expressed using a 3rd order polynomial as described below. After launch, coefficients have been revised using ground calibration points (GCP). But, the difference from the prelaunch values is very small.

$\theta_{\mathrm{atc}}=p_{x 0}+p_{x 1}\left(n-n_{c}\right)+p_{x 2}\left(n-n_{c}\right)^{2}+p_{x 3}\left(n-n_{c}\right)^{3}$,

$\theta_{\mathrm{ctc}}=p_{y 0}+p_{y 1}\left(n-n_{c}\right)+p_{y 2}\left(n-n_{\mathrm{cm}}\right)^{2}+p_{y 3}\left(n-n_{c}\right)^{3}$.

In the above equations, $p$ are coefficients of the polynomials, $\theta_{\text {atc }}$ and $\theta_{\text {ctc }}$ are the AT and CT angles from the nadir, $\mathrm{n}$ is the pixel number and $\mathrm{n}_{c}$ is the center pixel number. In the TANSO-CAI Level 1B, 1B+, and 2 data, the geoloca- tion is corrected using the above equations and digital elevation model (DEM) data. Only Band 4 uses a 512-pixels InGaAs-detector and complementary metal oxide semiconductor (CMOS) readouts. Odd and even number pixels have two different directions of readouts respectively. The detector readout masks for even number pixels are not opaque enough and its field of view has been stretched. These anomalies are minimized by shifting the geolocation separately for the odd and the even pixels in the TANSO-CAI Level 1B, 1B+, and Level 2 products.

\section{Conclusions}

Almost three years have passed since launch, and the overall functions and performances are successful and they are well within design objectives. TANSO-FTS is operating regularly with almost $100 \%$ duty cycle and the Level 1 is processed and distributed to NIES, NASA, and ESA typically within $5 \mathrm{~h}$ after observation. No significant degradation of SNR and spectral resolution has been observed. A few anomalies were found onboard, but they have stabilized since. The Level 1 algorithms have been updated since launch in order to correct these anomalies. The algorithms include the quality information for higher level processing. Together with the Level 1B data and prelaunch calibration data, degradation correction factors have been determined. GOSAT continues its observations and has been providing well-calibrated high spectral resolution data.

Acknowledgements. The authors would like to thank the GOSAT science team and the operation team. We especially acknowledge Raphael Desbiens of ABB Inc.; Hank Revercomb and Robert Knuteson of University of Wisconsin; Annmarie Eldering, David Crisp, Charles Miller, Christian Frankenberg, Denis O'Brien, Tommy Taylor, and Paul Wennberg of NASA-ACOS team; Tatsuya Yokota, Hiroshi Watanabe, Nobuyuki Kikuchi, and Yukio Yoshida of NIES; Andre Butz of the Karlsruhe Institute of Technology; Atsushi Takeuchi of NEC Toshiba Space Systems Inc.; Mayumi Yoshida, Riko Higuchi, Nami Sekio, and Yasushi Mitomi of the Remote Sensing Technology Center of Japan; and Hiroshi Otake.

Edited by: I. Aben

\section{References}

Butz, A., Guerlet, S., Hasekamp, O., Schepers, D., Galli, A., Aben, I., Frankenberg, C., Hartmann, J.-M., Tran, H., Kuze, A., Keppel-Aleks, G., Toon, G., Wunch, D., Wennberg, P., Deutscher, N., Griffith, D., Macatangay, R., Messerschmidt, J., Notholt, J., and Warneke, T.: Toward accurate $\mathrm{CO}_{2}$ and $\mathrm{CH}_{4}$ observations from GOSAT, Geophys. Res. Lett., 38, L14812, doi:10.1029/2011GL047888, 2011.

Crisp, D., Fisher, B. M., O’Dell, C., Frankenberg, C., Basilio, R., Bösch, H., Brown, L. R., Castano, R., Connor, B., Deutscher, 
N. M., Eldering, A., Griffith, D., Gunson, M., Kuze, A., Mandrake, L., McDuffie, J., Messerschmidt, J., Miller, C. E., Morino, I., Natraj, V., Notholt, J., O’Brien, D. M., Oyafuso, F., Polonsky, I., Robinson, J., Salawitch, R., Sherlock, V., Smyth, M., Suto, H., Taylor, T. E., Thompson, D. R., Wennberg, P. O., Wunch, D., and Yung, Y. L.: The ACOS $\mathrm{CO}_{2}$ retrieval algorithm - Part II: Global $\mathrm{X}_{\mathrm{CO}_{2}}$ data characterization, Atmos. Meas. Tech., 5, 687707, doi:10.5194/amt-5-687-2012, 2012.

Frankenberg, C., Butz, A., and Toon, G. C.: Disentangling chlorophyll fluorescence from atmospheric scattering effects in $\mathrm{O}_{2} \mathrm{~A}$ band spectra of reflected sun light, Geophys. Res. Lett., 38, L03801, doi:10.1029/2010GL045896, 2011a.

Frankenberg, C., Fisher, J. B., Worden, J., Badgley, G., Saatchi, S. S., Lee, J.-E., Toon, G. C., Butz, A., Jung, M., Kuze, A., and Yokota, T.: New global observations of the terrestrial carbon cycle from GOSAT: Patterns of plant fluorescence with gross primary productivity, Geophys. Res. Lett., 38, L17706, doi:10.1029/2011GL048738, 2011b.

Guanter, L., Frankenberg, C., Dudhia, A., Lewis, P. E., GomezDans, J., Kuze, A., Suto, H., and Grainger, R. G.: Retrieval and global assessment of terrestrial chlorophyll fluorescence from GOSAT space measurements, Remote Sens. Environ., 121, 236251, 2012.

Ishida, H., Nakajima, T. Y., and Nakajima, T.: Development of an unbiased cloud detection algorithm for a spaceborne multispectral imager, J. Geophys. Res., 114, D07206, doi:10.1029/2008JD010710, 2009.

Ishida, H., Nakajima, T. Y., Yokota, T., Kikuchi, N., and Watanabe, H.: Investigation of GOSAT TANSO-CAI cloud screening ability through an intersatellite comparison. J. Appl. Meteor. Climatol., 50, 1571-1586, 2011.

Joiner, J., Yoshida, Y., Vasilkov, A. P., Yoshida, Y., Corp, L. A., and Middleton, E. M.: First observations of global and seasonal terrestrial chlorophyll fluorescence from space, Biogeosciences, 8, 637-651, doi:10.5194/bg-8-637-2011, 2011.

Joiner, J., Yoshida, Y., Vasilkov, A. P., Middleton, E. M., Campbell, P. K. E., Yoshida, Y., Kuze, A., and Corp, L. A.: Filling-in of near-infrared solar lines by terrestrial fluorescence and other geophysical effects: simulations and space-based observations from SCIAMACHY and GOSAT, Atmos. Meas. Tech., 5, 809-829, doi:10.5194/amt-5-809-2012, 2012.

Keppel-Aleks, G., Toon, G. C., Wennberg, P. O., and Deutscher, N. M.: Reducing the impact of source brightness fluctuations on spectra obtained by Fourier-transform spectrometry, Appl. Opt., 46, 4774-4779, 2007.

Knuteson, R. O., Revercomb, H. E., Best, F. A., Ciganovich, N. C., Dedecker, R. G. Dirkx, T. P., Ellington, S. C., Feltz, W. F., Garcia, R. K., Howell, H. B., Smith, W. L., Short, J. F., and Tobin, D. C.: Atmospheric Emitted Radiance Interferometer. Part II: Instrument Performance, J. Atmos. Ocean Technol., 21, 1777-1789, 2004.

Kuze, A., Suto, H., Nakajima, M., and Hamazaki, T.: Thermal and near infrared sensor for carbon observation Fourier-transform spectrometer on the Greenhouse Gases Observing Satellite for greenhouse gases monitoring, Appl. Opt., 48, 6716-6733, 2009.

Kuze, A., O’Brien, D. M., Taylor, T. E., Day, J. O., O’Dell, C., Kataoka, F., Yoshida, M., Mitomi, Y., Bruegge, C., Pollock, H., Basilio, R., Helmlinger, M., Matsunaga, T., Kawakami, S., Shiomi, K., Urabe, T., and Suto, H.: Vicarious calibra- tion of the GOSAT sensors using the Railroad Valley desert playa, IEEE Trans. Geosci. Remote Sens., 49, 1781-1795, doi:10.1109/TGRS.2010.2089527, 2011a.

Kuze, A., Suto, H., Shiomi, K., and Nakajima, M.: GOSAT TANSO Calibration and characterization of 2 years on orbit operation, ISTS, Proceedings of The 28th International Symposium on Space Technology and Science (ISTS), Okinawa, 2011b.

Kuze, A., Suto, H., Shiomi, K., and Nakajima, M: Lessons learned from GOSAT and improvements for the next mission, Hyperspectral Imaging and Sounding of the Environment, OSA Technical Digest (CD) (Optical Society of America, 2011), paper JMA3, 2011c.

Kuze, A., Bruegge, C., O'Brien, D. M., Knuteson, R. O., and Yates, E.: Vicarious calibration and validation campaign of the GOSAT sensors at Railroad Valley, AGU fall meeting Suppl., Abstract A42D-07, 2011d.

Kuze, A., Taylor, T. E., Bruegge, C., Kataoka, F., O’Brien, D. M., Crisp, D., Pollock, H., Helmlinger, M., Schwandner, F., Kawakami, S., Shiomi, K., Tanaka, T., Ohyama, H., Suto, H., Naito, M., Harada, M., Murooka, J., Takeda, T., Urabe, T., Mitomi, Y., and Inoue, M.: Long term vicarious calibrations of GOSAT sensors and their error reduction techniques, to be submitted, 2012.

O’Dell, C. W., Connor, B., Bösch, H., O’Brien, D., Frankenberg, C., Castano, R., Christi, M., Eldering, D., Fisher, B., Gunson, M., McDuffie, J., Miller, C. E., Natraj, V., Oyafuso, F., Polonsky, I., Smyth, M., Taylor, T., Toon, G. C., Wennberg, P. O., and Wunch, D.: The ACOS $\mathrm{CO}_{2}$ retrieval algorithm - Part 1: Description and validation against synthetic observations, Atmos. Meas. Tech., 5, 99-121, doi:10.5194/amt-5-99-2012, 2012.

Parker, R., Boesch, H., Cogan, A., Fraser, A., Feng, L., Palmer, P.I., Messerschmidt, J., Deutscher, N., Griffith, D. W. T., Notholt, J., Wennberg, P. O., and Wunch, D.: Methane observations from the Greenhouse Gases Observing SATellite: Comparison to groundbased TCCON data and model calculations, Geophys. Res. Lett., 38, L15807, doi:10.1029/2011GL047871, 2011.

Saitoh, N., Imasu, R., Ota, Y., and Niwa, Y.: $\mathrm{CO}_{2}$ retrieval algorithm for the thermal infrared spectra of the Greenhouse Gases Observing Satellite: potential of retrieving $\mathrm{CO}_{2}$ vertical profile from high-resolution FTS sensor, J. Geophys. Res. 114, D17305, doi:10.1029/2008JD011500, 2009.

Sakuma, F., Bruegge, C., Rider, D., Brown, D., Geier, S., Kawakami, S., and Kuze, A.: OCO-GOSAT preflight cross calibration experiment, IEEE Trans. Geosci. Remote Sens., 48, 585599, 2010.

Simon A. R.: Data processing pipelines tailored for imaging Fourier-transform spectrometers, University of Laval, 2008.

Suto, H. and Kuze, A.: Correction of scan-speed instability of TANSO-FTS on GOSAT, AGU fall meeting Suppl., Abstract A51C-0107, doi:10.1029/2008JD011500, 2010.

Suto, H., Kuze, A., Shiomi, K., and Nakajima, M.,: Updated Level1 processing after two-years operation of TANSO-FTS, Proc. SPIE, 8154, 81541A, doi:10.1117/12.893405, 2011a.

Suto, H., Frankenberg, C., Crisp, D., and Kuze, A.: Characterization and correction of non-linearity effect on oxygen spectra of TANSO-FTS onboard GOSAT, AGU fall meeting Suppl., Abstract A33C-0210, $2011 \mathrm{~b}$.

Tadono, T., Shimada, M., Murakami, H., and Takaku, J.: Calibration of PRISM and AVNIR-2 Onboard ALOS “Daichi”, IEEE Trans. 
Geosci. Remote Sens., 47, 4042-4050, 2009.

Tobin, D. C., Revercomb, H. E., Knuteson, R. O., Best, F. A., Smith, W. L., Ciganovich, N. N., Dedecker, R. G., Dutcher, S., Ellington, S. D., Raymond K., Garcia, H., Howell, B., LaPorte, D. D., Mango, S. A., Pagano, T. S., Taylor, J. K., Delst, P., Vinson, K. H., and Werner, M. W.: Radiometric and spectral validation of Atmospheric Infrared Sounder observations with the aircraftbased Scanning High-Resolution Interferometer Sounder, J. Geophys. Res. 111, D09S02, doi:10.1029/2005JD006094, 2006.
Yoshida, Y., Ota, Y., Eguchi, N., Kikuchi, N., Nobuta, K., Tran, H., Morino, I., and Yokota, T.: Retrieval algorithm for $\mathrm{CO}_{2}$ and $\mathrm{CH}_{4}$ column abundances from short-wavelength infrared spectral observations by the Greenhouse gases observing satellite, Atmos. Meas. Tech., 4, 717-734, doi:10.5194/amt-4-717-2011, 2011. 